\title{
Viscous cosmology in new holographic dark energy model and the cosmic acceleration
}

\author{
C. P. Singh ${ }^{\mathrm{a}}$, Milan Srivastava ${ }^{\mathrm{b}}$ \\ Department of Applied Mathematics, Delhi Technological University, Bawana Road, Delhi 110 042, India
}

Received: 19 January 2018 / Accepted: 27 February 2018 / Published online: 6 March 2018

(C) The Author(s) 2018. This article is an open access publication

\begin{abstract}
In this work, we study a flat FriedmannRobertson-Walker universe filled with dark matter and viscous new holographic dark energy. We present four possible solutions of the model depending on the choice of the viscous term. We obtain the evolution of the cosmological quantities such as scale factor, deceleration parameter and transition redshift to observe the effect of viscosity in the evolution. We also emphasis upon the two independent geometrical diagnostics for our model, namely the statefinder and the $\mathrm{Om}$ diagnostics. In the first case we study new holographic dark energy model without viscous and obtain power-law expansion of the universe which gives constant deceleration parameter and statefinder parameters. In the limit of the parameter, the model approaches to $\Lambda C D M$ model. In new holographic dark energy model with viscous, the bulk viscous coefficient is assumed as $\zeta=\zeta_{0}+\zeta_{1} H$, where $\zeta_{0}$ and $\zeta_{1}$ are constants, and $H$ is the Hubble parameter. In this model, we obtain all possible solutions with viscous term and analyze the expansion history of the universe. We draw the evolution graphs of the scale factor and deceleration parameter. It is observed that the universe transits from deceleration to acceleration for small values of $\zeta$ in late time. However, it accelerates very fast from the beginning for large values of $\zeta$. By illustrating the evolutionary trajectories in $r-s$ and $r-q$ planes, we find that our model behaves as an quintessence like for small values of viscous coefficient and a Chaplygin gas like for large values of bulk viscous coefficient at early stage. However, model has close resemblance to that of the $\Lambda C D M$ cosmology in late time. The $\mathrm{Om}$ has positive and negative curvatures for phantom and quintessence models, respectively depending on $\zeta$. Our study shows that the bulk viscosity plays very important role in the expansion history of the universe.
\end{abstract}

\footnotetext{
a e-mail: cpsphd@ rediffmail.com

be-mail: milandtu@gmail.com
}

\section{Introduction}

The astrophysical data obtained from high redshift surveys of supernovae [1-3], Wilkinson Microwave Anisotropy Probe (WMAP) [4,5] and the large scale structure from Slogan Digital Sky Survey (SDSS) [6,7] support the existence of dark energy (DE). The DE is considered as an exotic energy component with negative pressure. The cosmological analysis of these observations suggest that the universe consists of about $70 \% \mathrm{DE}, 30 \%$ dust matter (cold dark matter plus baryons), and negligible radiation. It is the most accepted idea that DE leads to the late-time accelerated expansion of the universe. Nevertheless, the nature of such a DE is still the source of debate. Many theoretical models have been proposed to describe this late-time acceleration of the universe. The most obvious theoretical candidate for DE is the cosmological constant [8], which has the equation of state (EoS) $\omega_{\Lambda}=-1$. However, it suffers the so-called cosmological constant (CC) problem (the fine-tuning problem) and the cosmic coincidence problem [9-11]. Both of these problems are related to the DE density.

In order to solve the cosmological constant problems, many candidates such as quintessence [12,13], phantom [14], tachyon field [15], quintom [16], holographic dark energy $[17,18]$, agegraphic dark energy $[19,20]$ have been proposed to explain the nature of DE phenomenon. Starobinsky [21] and Kerner et al. [22] proposed an another way to explain the accelerated expansion of the universe by modifying the geometrical part of Einstein field equations which is known as modified gravity theory.

In recent years, the considerable interest has been noticed in the study of holographic dark energy (HDE) model to explain the recent phase transition of the universe. The idea of HDE is basically based on the holographic principle [23-25]. According to holographic principle a short distance (ultraviolet) cut-off $\Lambda$ is related to the long distance (infrared) cut-off $L$ due to the limit set by the formation of a black hole [26]. Li 
[18] argued that the total energy in a region of size $L$ should not exceed the mass of a black hole of same size for a system with ultraviolet (UV) cut-off $\Lambda$, thus $L^{3} \rho_{\Lambda} \leq L M_{p l}^{2}$, where $\rho_{\Lambda}$ is the quantum zero-point energy density caused by UV cot-off $\Lambda$ and $M_{p l}$ is the reduced Planck mass $M_{p l}^{-2}=8 \pi G$. The largest $L$ allowed is the one saturating this inequality, thus the HDE density is defined as $\rho_{\Lambda}=3 c^{2} M_{p l}^{2} L^{-2}$, where $c$ is a numerical constant. The UV cut-off is related to the vacuum energy, and the infrared (IR) cut-off is related to the large scale structure of the universe, i.e., Hubble horizon, particle horizon, event horizon, Ricci scalar, etc. The HDE model suffers the choice of IR cut-off problem. In the Ref. [17], it has been discussed that the HDE model with Hubble horizon or particle horizon can not drive the accelerated expansion of the universe. However, HDE model with event horizon can drive the accelerated expansion of the universe [18]. The drawback with event horizon is that it is a global concept of spacetime and existence of universe, depends on the future evolution of the universe. The HDE with event horizon is also not compatible with the age of some old high redshift objects [27]. Gao et al. [28] proposed IR cut-off as a function of Ricci scalar. So, the length $L$ is given by the average radius of Ricci scalar curvature.

As the origin of the HDE is still unknown, Granda and Oliveros [29] proposed a new IR cut-off for HDE, known as new holographic dark energy (NHDE), which besides the square of the Hubble scale also contains the time derivative of the Hubble scale. The advantage is that this NHDE model depends on local quantities and avoids the causality problem appearing with event horizon IR cut-off. The authors, in their other paper [30], reconstructed the scalar field models for HDE by using this new IR cut-off in flat FriedmannRobertson-Walker (FRW) universe with only DE content. Karami and Fehri [31] generated the results of Ref. [29] for non-flat FRW universe. Malekjani et al. [32] have studied the statefinder diagnostic with new IR cut-off proposed in [29] in a non-flat model. Sharif and Jawad [33] have investigated interacting NHDE model in non-flat universe. Debnath and Chattopadhyay [34] have considered flat FRW model filled with mixture of dark matter and NHDE, and have studied the statefinder and $\mathrm{Om}$ diagnostics. Wang and $\mathrm{Xu}$ [35] have obtained the constraints on HDE model with new IR cut-off via the Markov Chain Monte Carlo method with the combined constraints of current cosmological observations. Oliveros and Acero [36] have studied NHDE model with a non-linear interaction between the DE and dark matter (DM) in flat FRW universe.

A large number of models within modified theories can explain the DE phenomenon. It is therefore important to find the ways to discriminate among various competing models. For this purpose, Sahni et al. [37] and Alam et al. [38] introduced an important geometrical diagnostic, known as statefinder pair $\{r, s\}$ to remove the degeneracy of $H_{0}$ and $q_{0}$ of different DE models. The statefinder diagnostic has been extensively used in the literature to distinguish among various models of DE and modified theories of gravity. The various DE models have different evolutionary trajectories in $(r, s)$ plane.

In order to complement the statefinder [37,38], a new diagnostic called $O m$ was proposed by Sahni et al. [39] in 2008, which is used to distinguish among the energy densities of various DE models. The advantage of Om over the statefinder parameters is that, $O m$ involves only the first order derivative of scale factor. For the $\Lambda C D M$ model $O m$ diagnostic turns out to be constant. We provide the mathematical expressions of statefinder and $\mathrm{Om}$ diagnostic in the appropriate section.

Evolution of the universe involves a sequence of dissipative processes. These processes include bulk viscosity, shear viscosity and heat transport. The theory of dissipation was proposed by Eckart [40] and the full causal theory was developed by Israel and Stewart [41]. In the case of isotropic and homogeneous model, the dissipative process is modeled as a bulk viscosity, see Refs. [42-50]. Brevik et al. [51] discussed the general account about viscous cosmology for early and late time universe. Norman and Brevik [52] analyze characteristic properties of two different viscous cosmology models for the future universe. In other paper, Norman and Brevik [53] derived a general formalism for bulk viscous and estimated the bulk viscosity in the cosmic fluid. The HDE model has been studied in some recent literatures $[54,55]$ under the influence of bulk viscosity. Feng and Li [56] have investigated the viscous Ricci dark energy (RDE) model by assuming that there is bulk viscosity in the linear barotropic fluid and RDE. Singh and Kumar [57] have discussed the statefinder diagnosis of the viscous HDE cosmology. The main motive of this work is to explain the acceleration with the help of bulk viscosity for new holographic dark energy (NHDE) in GR which has not been studied sofar.

The bulk viscosity introduces dissipation by only redefining the effective pressure, $p_{e f f}$, according as $p_{\text {eff }}=p-$ $3 \zeta H$, where $\zeta$ is the bulk viscosity coefficient and $H$ is the Hubble parameter. In this paper, we are interested when the universe is dominated by viscous HDE and dark matter with Granda-Oliveros IR cut-off to study the influence of bulk viscosity to the cosmic evolution. We consider the general form of bulk viscosity $\zeta=\zeta_{0}+\zeta_{1} H$, where $\zeta_{0}$ and $\zeta_{1}$ are the constants and $H$ is the Hubble's parameter, see Refs. [42,43]. First, we discuss the non-viscous NHDE model to find out the exact solution of the field equations. In the second case, we find out the exact solutions of the field equations with constant and varying bulk viscous term. We find the exact forms of scale factor, deceleration parameter and transition redshift and discuss the evolution through the graphs. We also discuss the geometrical diagnostics like statefinder parameter and $\mathrm{Om}$ diagnostic to discriminate our model with $\Lambda C D M$. We plot the trajectories 
of these parameters and observe the effect of bulk viscous coefficient.

The paper is organised as follows. In Sect. 2, we discuss the non-viscous HDE model with new IR cut-off. Section 3 presents the viscous NHDE model and is divided into Sects. 3.1 and 3.2. In Sects. 3.1 and 3.2 we present the solutions with constant and time varying bulk viscous term. Section 4 presents the summary of the results.

\section{Non-viscous NHDE model}

We consider a spatially homogeneous and isotropic flat Friedmann-Robertson-Walker (FRW) space-time, specified by the line element

$d s^{2}=d t^{2}-a^{2}(t)\left[d r^{2}+r^{2}\left(d \theta^{2}+\sin ^{2} \theta d \phi^{2}\right)\right]$,

where $a(t)$ is the scale factor, $t$ is the cosmic time and $(r, \theta, \phi)$ are the comoving coordinates.

We consider that the Universe is filled with NHDE plus pressureless dark matter (DM) (ignoring the contribution of the baryonic matter here for simplicity). For Einstein field equations $R_{\mu \nu}-g_{\mu \nu} R / 2=T_{\mu \nu}$ in the units where $8 \pi G=$ $c=1$, we obtain the Friedmann equations for the metric (1) as

$$
\begin{aligned}
3 H^{2} & =\rho_{m}+\rho_{d}, \\
2 \dot{H}+3 H^{2} & =-p_{d},
\end{aligned}
$$

where $\rho_{m}$ and $\rho_{d}$ are the energy density of DM and NHDE, respectively, and $p_{d}$ is the pressure of the NHDE. A relation between $\rho_{d}$ and $p_{d}$ is given by equation of state (EoS) parameter $\omega_{d}=p_{d} / \rho_{d}$. Here, $H=\dot{a} / a$ is the Hubble parameter. A dot denotes a derivative with respect to the cosmic time $t$.

As suggested by Granda and Oliveros in paper [29], the energy density of HDE with the new IR cut-off is given by

$\rho_{d}=3\left(\alpha H^{2}+\beta \dot{H}\right)$,

where $\alpha$ and $\beta$ are the dimensionless parameters, which must satisfy the restrictions imposed by the current observational data.

Using (4), Eqs. (2) and (3) give

$\dot{H}+\frac{3\left(1+\alpha \omega_{d}\right)}{\left(2+3 \beta \omega_{d}\right)} H^{2}=0$.

The solution of (5) is given by

$H=\frac{1}{c_{0}+\frac{3\left(1+\alpha \omega_{d}\right)}{\left(2+3 \beta \omega_{d}\right)} t}$,

where $c_{0}$ is an integration constant. Equation (6) can be rewritten as

$H=\frac{H_{0}}{\left\{1+\frac{3 H_{0}\left(1+\alpha \omega_{d}\right)}{\left(2+3 \beta \omega_{d}\right)}\left(t-t_{0}\right)\right\}}$, where $H_{0}$ is the present value of the Hubble parameter at $t=$ $t_{0}$, where NHDE starts to dominate. As we know $H=\dot{a} / a$, Eq. (7) gives the solution for the scale factor which is given by

$$
\begin{gathered}
a=a_{0}\left\{1+\frac{3\left(1+\alpha \omega_{d}\right) H_{0}}{\left(2+3 \beta \omega_{d}\right)}\left(t-t_{0}\right)\right\}^{\frac{\left(2+3 \beta \omega_{d}\right)}{3\left(1+\alpha \omega_{d}\right)}}, \\
\text { for } \alpha \neq-1 / \omega_{d}, \beta \neq-2 / 3 \omega_{d},
\end{gathered}
$$

where $a_{0}$ is the present value of the scale factor at a cosmic time $t=t_{0}$. Equation (8) shows the power-law $a \propto t^{m}$, where $m$ is a constant, type expansion of the scale factor. As we know that the universe will undergo with decelerated expansion for $m<1$, i.e., $\left(2+3 \beta \omega_{d}\right)<\left(3+3 \alpha \omega_{d}\right)$ in our case whereas it accelerates for $m>1$, i.e., $\left(2+3 \beta \omega_{d}\right)>$ $\left(3+3 \alpha \omega_{d}\right)$. For $m=1$, i.e., $\left(2+3 \beta \omega_{d}\right)=\left(3+3 \alpha \omega_{d}\right)$, the universe will show marginal inflation. In the absence of NHDE, i.e., for $\alpha=\beta=0$, we get the dark matter dominated scale factor, $a=a_{0}\left(1+\frac{3}{2} H_{0}\left(t-t_{0}\right)\right)^{2 / 3}$.

Let us consider the deceleration parameter (DP) which is very useful parameter to discuss the behaviour of the universe. The sign (positive or negative) of DP explains whether the universe decelerates or accelerates. It is defined as $q=-\frac{a \ddot{a}}{\dot{a}^{2}}$. From (8), we get

$q=\frac{3\left(1+\alpha \omega_{d}\right)}{\left(2+3 \beta \omega_{d}\right)}-1$,

which is a constant value throughout the evolution of the universe. The universe will expand with decelerated rate for $q>0$, i.e., $\left(2+3 \beta \omega_{d}\right)<\left(3+3 \alpha \omega_{d}\right)$, accelerated rate for $q<0$, i.e., $\left(2+3 \beta \omega_{d}\right)>\left(3+3 \alpha \omega_{d}\right)$ and marginal inflation for $q=0$, i.e., $\left(2+3 \beta \omega_{d}\right)=\left(3+3 \alpha \omega_{d}\right)$. One can explicitly observe the dependence of DP $q$ on the model parameters $\alpha, \beta$ and EoS parameter $\omega_{d}$ under above constraints. Thus, we can obtain a decelerated or accelerated expansion of the universe depending on the suitable choices of these parameters. In this case, the model does not show the phase transition due to power-law expansion or constant DP. The model shows marginal inflation, $q=0$ when $\omega_{d}=1 / 3(\beta-\alpha)$. Using Markov chain Monte Carlo method on latest observational data, Wang and $\mathrm{Xu}$ [35] have constrained the NHDE model and obtained the best fit values of the parameters $\alpha=$ $0.8502_{-0.0875-0.1064}^{+0.0984+0.1299}$ and $\beta=0.4817_{-0.0773-0.0955}^{+0.0842+0.1176}$ with $1 \sigma$ and $2 \sigma$ errors in flat model. In the best fit NHDE models, they have obtained the EoS parameter $\omega_{d}=-1.1414 \pm 0.0608$. Putting these values of parameters (excluding the errors) in Eq.(9), we get $q=-0.7468$, which shows that our NHDE model is consistent with current observation data given in [35].

In order to discriminate among the various DE models, Sahni et al. [37] and Alam et al. [38] introduced a new geometrical diagnostic pair for DE, which is known as statefinder pair and is denoted as $\{r, s\}$. The statefinder probes the expan- 
sion dynamics of the universe through higher derivatives of the scale factor and is a geometrical diagnosis in the sense that it depends on the scale factor and hence describes the spacetime. The statefinder pair is defined as

$r=\frac{\dddot{a}}{a H^{3}} \quad$ and $\quad s=\frac{r-1}{3(q-1 / 2)}$.

Substituting the required values from (8) and (9) into (10), we get

$r=1-\frac{9\left(1+\alpha \omega_{d}\right)}{\left(2+3 \beta \omega_{d}\right)}+\frac{18\left(1+\alpha \omega_{d}\right)^{2}}{\left(2+3 \beta \omega_{d}\right)^{2}}$,

and

$s=\frac{2\left(1+\alpha \omega_{d}\right)}{2+3 \beta \omega_{d}}$.

From (11) and (12), we can observe that these statefinder parameters are constant whose values depend on $\alpha, \beta$ and $\omega_{d}$. As Sahni et al. [37] and Alam et al. [38] have observed that Lambda cold dark matter $(\Lambda \mathrm{CDM})$ model and standard cold dark matter (SCDM) model have fixed point values of statefinder parameter $\{r, s\}=\{1,0\}$ and $\{r, s\}=\{1,1\}$, respectively. Putting the values of parameters [35] as mentioned above, we observe that this set of data do not favor the NHDE model over the $\Lambda$ CDM as well as $S C D M$ model. However, NHDE model behaves like $S C D M$ model for $\alpha=3 \beta / 2$. We can also observe that this model approaches to $\{r, s\} \rightarrow\{1,0\}$ in the limit of $\alpha \rightarrow-1 / \omega_{d}$ but there is no such value of parameters which would clearly show the $\Lambda \mathrm{CDM}$.

\section{Viscous NHDE model}

In the previous section, we have observed that the nonviscous NHDE model gives constant DP which is unable to represent the phase transition. However, the observations show that the phase transition plays a vital role in describing the evolution of the universe. Therefore, it will be interesting to study the NHDE model with viscous to investigate whether a viscous NHDE model with Granda-Oliveros IR cut-off would be able to find the phase transition.

In an isotropic and homogeneous FRW universe, the dissipative effects arise due to the presence of bulk viscosity in cosmic fluids as shear viscosity plays no role. DE with bulk viscosity has a peculiar property to cause accelerated expansion of phantom type in the late time evolution of the universe [58-60]. It can also alleviate the problem like age problem and coincidence problem.

Let us assume that the effective pressure of NHDE is a sum of pressure of NHDE and bulk viscosity, i.e., the universe is filled with bulk viscous NHDE plus pressureless dark matter (DM) (ignoring the contribution of the baryonic matter here for simplicity). Then, the field equations (2) and (3) modify to

$3 H^{2}=\rho_{m}+\rho_{d}$,

$2 \dot{H}+3 H^{2}=-\tilde{p_{d}}$,

where $\tilde{p}_{d}=p_{d}-3 H \zeta$ is the effective pressure of NHDE. This form of effective pressure was originally proposed by Eckart [40] in the context of relativistic dissipative process occurring in thermodynamic systems went out of local thermal equilibrium. The term $\zeta$ is the bulk viscosity coefficient [61-63]. On the thermodynamical grounds, $\zeta$ is conventionally chosen to be a positive quantity and generically depends on the cosmic time $t$, or redshift $z$, or the scale factor $a$, or the energy density $\rho_{d}$, or a more complicated combination form. Maartens [64] assumed the bulk viscous coefficient as $\zeta \propto \rho^{n}$, where $n$ is a constant. In the Refs. [42-44], the most general form of bulk viscosity has been considered with generalized equation of state. Following [42-44,65], we take the bulk viscosity coefficient in the following form.

$\zeta=\zeta_{0}+\zeta_{1} H$

where $\zeta_{0}$ and $\zeta_{1}$ are positive constants. The motivation for considering this bulk viscosity has been discussed in Refs. [42-44].

From the dynamical equations (13) and (14), we can formulate a first order differential equation for the Hubble parameter by using Eqs. (4) and (15) as,

$\dot{H}+\frac{3\left(1+\alpha \omega_{d}\right)}{\left(2+3 \beta \omega_{d}\right)} H^{2}-\frac{3 \zeta}{\left(2+3 \beta \omega_{d}\right)} H=0$.

It can be observed that Eq. (16) reduces to the non-viscous equation (5) for $\zeta=0$ as discussed in previous section.

In the following subsections, we classify different viscous NHDE models arises due to the constant and variable bulk viscous coefficient. We analyze the behavior of the scale factor, DP, statefinder parameter and $\mathrm{Om}$ diagnostic of these different cases.

\subsection{NHDE Model with constant bulk viscosity}

The simplest case of viscous NHDE model is to be taken with constant bulk viscous coefficient. Therefore, assuming $\zeta_{1}=0$ in Eq. (15), the bulk viscous coefficient reduces to

$\zeta=\zeta_{0}=$ const.

Using (17) into (16), we get

$\dot{H}+\frac{3\left(1+\alpha \omega_{d}\right)}{\left(2+3 \beta \omega_{d}\right)} H^{2}-\frac{3 \zeta_{0}}{\left(2+3 \beta \omega_{d}\right)} H=0$. 
The solution of (18) in terms of cosmic time $t$ can be given by

$H=e^{\frac{3 \zeta_{0} t}{\left(2+3 \beta \omega_{d}\right)}}\left[c_{1}+\frac{\left(1+\alpha \omega_{d}\right)}{\zeta_{0}} e^{\frac{3 \zeta_{0} t}{\left(2+3 \beta \omega_{d}\right)}}\right]^{-1}$,

where $c_{1}$ is the constant of integration. From (19), we get the evolution of the scale factor as

$a=c_{2}\left[c_{1}+\frac{\left(1+\alpha \omega_{d}\right)}{\zeta_{0}} e^{\frac{3 \zeta_{0} t}{\left(2+3 \beta \omega_{d}\right)}}\right]^{\frac{\left(2+3 \beta \omega_{d}\right)}{3\left(1+\alpha \omega_{d}\right)}}$,

where $c_{2}$ is an integration constant. The above scale factor can be rewritten as

$a=\left[1+\frac{H_{0}\left(1+\alpha \omega_{d}\right)}{\zeta_{0}}\left\{e^{\frac{3 \zeta_{0}\left(t-t_{0}\right)}{\left(2+3 \beta \omega_{d}\right)}}-1\right\}\right]^{\frac{\left(2+3 \beta \omega_{d}\right)}{3\left(1+\alpha \omega_{d}\right)}}$,

$$
\text { for } \alpha \neq-1 / \omega_{d}, \zeta_{0} \neq 0
$$

where $t_{0}$ is the present cosmic time. Here, we get exponential form of the scale factor which shows non-singular solution. Equation (21) shows that in early stages of the evolution, the scale factor can be approximated as $a(t) \sim$ $\left[1+\frac{3 H_{0}\left(1+\alpha \omega_{d}\right)}{\left(2+3 \beta \omega_{d}\right)}\left(t-t_{0}\right)\right]^{\frac{\left(2+3 \beta \omega_{d}\right)}{3\left(1+\alpha \omega_{d}\right)}}$, and as $\left(t-t_{0}\right) \rightarrow \infty$, the scale factor approaches to a form like that of the de Sitter universe, i.e., $a(t) \rightarrow \exp \left[\frac{3 \zeta_{0}\left(t-t_{0}\right)}{\left(2+3 \beta \omega_{d}\right)}\right]$. Thus, we observe that the universe starts with a finite volume followed by an early decelerated epoch, then making a transition into the accelerated epoch in the late time of the evolution.

From (21), we can obtain the Hubble parameter in terms of scale factor $a$ as

$$
\begin{aligned}
H(a)= & \frac{H_{0}}{\left(1+\alpha \omega_{d}\right)} \\
& \times\left[\frac{\zeta_{0}}{H_{0}}+\left\{\left(1+\alpha \omega_{d}\right)-\frac{\zeta 0}{H_{0}}\right\} a^{-\frac{\left(3+3 \alpha \omega_{d}\right)}{\left(2+3 \beta \omega_{d}\right)}}\right],
\end{aligned}
$$

where $H_{0}$ is the present value of the Hubble parameter and we have made the assumption that the present value of scale factor is $a_{0}=1$. The derivative of $\dot{a}$ with respect to $a$ can be obtained as [65]

$$
\begin{aligned}
\frac{d \dot{a}}{d a}= & \frac{H_{0}}{\left(1+\alpha w_{d}\right)}\left[\frac{\zeta_{0}}{H_{0}}-\left\{\left(1+\alpha w_{d}\right)-\frac{\zeta_{0}}{H_{0}}\right\}\right. \\
& \left.\times\left(\frac{\left(1+3(\alpha-\beta) w_{d}\right)}{2+3 \beta w_{d}}\right) a^{-\frac{3\left(1+\alpha w_{d}\right)}{\left(2+3 \beta w_{d}\right)}}\right] .
\end{aligned}
$$

Equation (23) to zero, the transition scale factor $a_{T}$ can be obtained as

$a_{T}=\left[\frac{\left(1+3(\alpha-\beta) w_{d}\right)\left\{\left(1+\alpha w_{d}\right) H_{0}-\zeta_{0}\right\}}{\left(2+3 \beta w_{d}\right) \zeta_{0}}\right]^{\frac{\left(2+3 \beta w_{d}\right)}{3\left(1+\alpha w_{d}\right)}}$.

The corresponding transition redshift $z_{T}$, where $a=(1+$ $z)^{-1}$, is

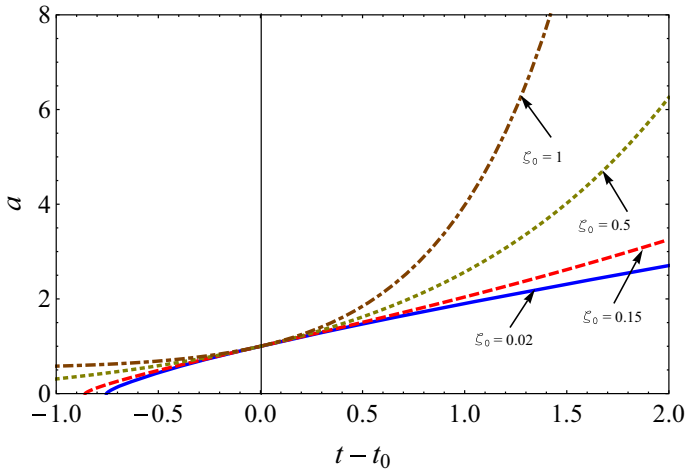

Fig. 1 The scale factor evolution for different values of $\zeta_{0}>0$ with $H_{0}=1, \omega_{d}=-0.5, \alpha=0.8502$ and $\beta=0.4817$

$z_{T}=\left[\frac{\left(1+3(\alpha-\beta) w_{d}\right)\left\{\left(1+\alpha w_{d}\right) H_{0}-\zeta_{0}\right\}}{\left(2+3 \beta w_{d}\right) \zeta_{0}}\right]^{-\frac{\left(2+3 \beta w_{d}\right)}{3\left(1+\alpha w_{d}\right)}}-1$.

From (24) or (25), we observe that for $\zeta_{0}=\frac{\left\{1+3(\alpha-\beta) w_{d}\right\} H_{0}}{3}$, the transition from decelerated phase to accelerated phase occurs at $a_{T}=1$ or $z_{T}=0$, which corresponds to the present time of the universe. On taking the observed values of $\alpha=0.8502$ and $\beta=0.4817$ [35], $H_{0}=1$ and $\omega_{d}=-0.5$ in this expression of $\zeta_{0}$, we get $\zeta_{0}=0.15$. Figure 1 represents the evolution of the scale factor $a(t)$ with respect to time $\left(t-t_{0}\right)$ for different values of $\zeta_{0}>0$. It is observed that the transition from decelerated to accelerated phase takes place in late time for small values of $\zeta_{0}$, i.e., in $0<\zeta_{0}<0.15$. The transition from decelerated phase to accelerated one occurs at $a_{T}=1$ for $\zeta_{0}=0.15$ which corresponds to the present time of the universe. However, the transition takes place in early stages of the evolution for large values of $\zeta_{0}$, i.e., $\zeta_{0}>0.15$. Thus, as the value of $\zeta_{0}$ increases, the scale factor expands more rapidly with exponential rate.

The result regarding the transition of the universe into the accelerated epoch discussed above can be further verified by studying the evolution of DP $q$. In this case, DP is given by

$q=\frac{3\left\{\left(1+\alpha \omega_{d}\right)-\frac{\zeta_{0}}{H_{0}}\right\}}{\left(2+3 \beta \omega_{d}\right)} e^{-\frac{3 \zeta_{0}\left(t-t_{0}\right)}{\left(2+3 \beta \omega_{d}\right)}}-1$.

Thus, we find a time-varying DP in the case of constant viscous NHDE, which describes the phase transition of the evolution of the universe. DP must change its sign at $t=t_{0}$, i.e., the time at which the viscous NHDE begins to dominate. This time can be achieved if $\left[1+3(\alpha-\beta) \omega_{d}\right] H_{0}=3 \zeta_{0}$. The universe must decelerate for $t<t_{0}$ and accelerate for $t>t_{0}$ for any parametric values of $\alpha, \beta$ and $\omega_{d}$.

From (26), DP can be written in terms of scale factor as 


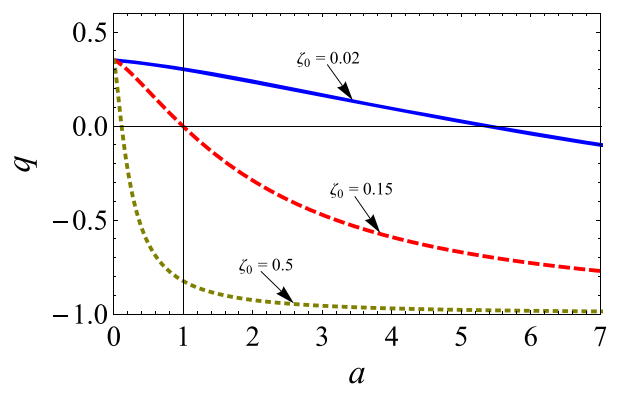

(a)

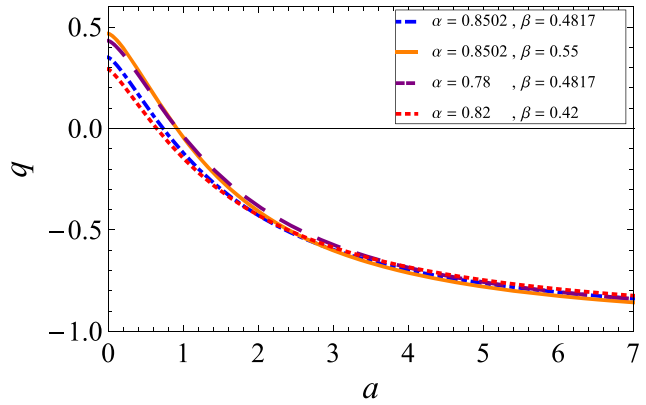

(b)

Fig. 2 a The variation of $q$ with $a$ for different values of $\zeta_{0}$ with fixed $\omega_{d}=-0.5, \alpha=0.8502$ and $\beta=0.4817$. b The variation of $q$ with $a$ for different values of $\alpha$ and $\beta$ with fixed $\zeta_{0}=0.2$ and $\omega_{d}=-0.5$

$$
\begin{aligned}
q= & \frac{\left\{3\left(1+\alpha \omega_{d}\right)-3 \zeta_{0}\right\}}{\left(2+3 \beta \omega_{d}\right)} \\
& \times\left[\frac{\left(1+\alpha \omega_{d}\right)}{\left(a^{\frac{3+3 \alpha \omega_{d}}{2+3 \beta \omega_{d}}}-1\right) \zeta_{0}+\left(1+\alpha \omega_{d}\right)}\right]-1 .
\end{aligned}
$$

In terms of red shift $z$, the above equation becomes

$$
\begin{aligned}
q= & \frac{\left\{3\left(1+\alpha \omega_{d}\right)-3 \zeta_{0}\right\}}{\left(2+3 \beta \omega_{d}\right)} \\
& \times\left[\frac{\left(1+\alpha \omega_{d}\right)}{\left((1+z)^{-\frac{3+3 \alpha \omega_{d}}{2+3 \beta \omega_{d}}}-1\right) \zeta_{0}+\left(1+\alpha \omega_{d}\right)}\right]-1 .
\end{aligned}
$$

When the bulk viscous parameter and all other parameters are zero, the deceleration parameter $q=1 / 2$, which corresponds to a decelerating matter-dominated universe with null bulk viscosity. However, when only the bulk viscous term $\zeta_{0}=0$, the value of $q$ is same as obtained in Eq. (9) for non-viscous NHDE model.

The present value of $q$ corresponds to $z=0$ or $a=1$ is,

$q_{0}=q(a=1)=\frac{3\left(1+\alpha \omega_{d}\right)-3 \zeta_{0}}{\left(2+3 \beta \omega_{d}\right)}-1$.

This equation shows that if $3 \zeta_{0}=\left[1+3(\alpha-\beta) \omega_{d}\right]$, the deceleration parameter $q_{0}=0$. This implies that the transition into the accelerating phase would occur at the present time. The current DP $q_{0}<0$ if $3 \zeta_{0}>\left[1+3(\alpha-\beta) \omega_{d}\right]$, implying that the present universe is in the accelerating epoch and it entered this epoch at an early stage. But $q_{0}>0$ if $3 \zeta_{0}<\left[1+3(\alpha-\beta) \omega_{d}\right]$ implying that the present universe is decelerating and it will be entering the accelerating phase at a future time. The evolution of $q$ with $a$ is shown in Fig. 2 by taking fixed constant $\alpha$ and $\beta$ (or $\zeta_{0}$ ), from which we can see that the evolution of the universe is from deceleration to acceleration. Figure $2 \mathrm{a}$ illustrates the evolutionary history of DP for different value of $\zeta_{0}$ with $\omega_{d}=-0.5, \alpha=0.8502$ and $\beta=0.4817$. On considering $\alpha=0.8502, \beta=0.4817$ [35] and $\omega_{d}=-0.5$ in Eq. (29), we get $\zeta_{0}=0.15$ which gives $q_{0}=0$. Thus, the transition into accelerating phase would occur at present time. If $\zeta_{0}>0.15, q_{0}<0$, i.e., the universe is in accelerating epoch and it entered this epoch at an early stage. If $\zeta_{0}<0.15, q_{0}>0$, i.e., the universe is in decelerating epoch and it enters in an accelerating phase in future. Thus, the larger the value $\zeta_{0}$ is, the earlier acceleration occurs. The similar results for a fixed $\zeta_{0}$ also appear in Fig. 2b. The larger the values $\alpha$ and $\beta$ is, the earlier $q$ changes it sign from $q>0$ to $q<0$ for a fixed $\zeta_{0}$. In both Fig. 2a, b, we observe that $q \rightarrow-1$ in late time of evolution.

Statefinder diagnostic

From above discussion we conclude that there is a transition from decelerated phase to accelerated one in future for small bulk viscous coefficient, $0<\zeta_{0}<0.15$. It takes place to the present time for $\zeta=0.15$. However, the transition takes place in past for $\zeta>0.15$. The behavior of scale factor and deceleration parameter shows that the constant bulk viscous coefficient plays the role of DE. In what follows, we will present the statefinder diagnostic of the viscous NHDE model . In this model, the statefinder parameters defined in (10) can be obtained as

$$
\begin{gathered}
r=1+\frac{9\left(\frac{\zeta_{0}}{H_{0}}-\left(1+\alpha \omega_{d}\right)\right)\left(1-\frac{1+\alpha \omega_{d}}{\left(2+3 \beta \omega_{d}\right)}\right)}{\left(2+3 \beta \omega_{d}\right)} e^{-\frac{3 \zeta_{0}\left(t-t_{0}\right)}{\left(2+3 \beta \omega_{d}\right)}} \\
+\frac{9\left(\frac{\zeta_{0}}{H_{0}}-\left(1+\alpha \omega_{d}\right)\right)^{2}}{\left(2+3 \beta \omega_{d}\right)^{2}} e^{-\frac{6 \zeta_{0}\left(t-t_{0}\right)}{\left(2+3 \beta \omega_{d}\right)}}
\end{gathered}
$$

and

$$
s=\frac{\frac{2\left(\frac{\zeta_{0}}{H_{0}}-\left(1+\alpha \omega_{d}\right)\right)\left(1-\frac{1+\alpha \omega_{d}}{\left(2+3 \beta \omega_{d}\right)}\right)}{\left(2+3 \beta \omega_{d}\right)} e^{-\frac{3 \zeta_{0}\left(t-t_{0}\right)}{\left(2+3 \beta \omega_{d}\right)}}+\frac{2\left(\frac{\zeta_{0}}{H_{0}}-\left(1+\alpha \omega_{d}\right)\right)^{2}}{\left(2+3 \beta \omega_{d}\right)^{2}} e^{-\frac{6 \zeta_{0}\left(t-t_{0}\right)}{\left(2+3 \beta \omega_{d}\right)}}}{\frac{2\left(\left(1+\alpha \omega_{d}\right)-\frac{\zeta_{0}}{H_{0}}\right)}{\left(2+3 \beta \omega_{d}\right)} e^{-\frac{3 \zeta_{0}\left(t-t_{0}\right)}{\left(2+3 \beta \omega_{d}\right)}}-1} .
$$

Here, these values of statefinder parameter are timedependent and this is due to the bulk viscous coefficient $\zeta_{0}$. 


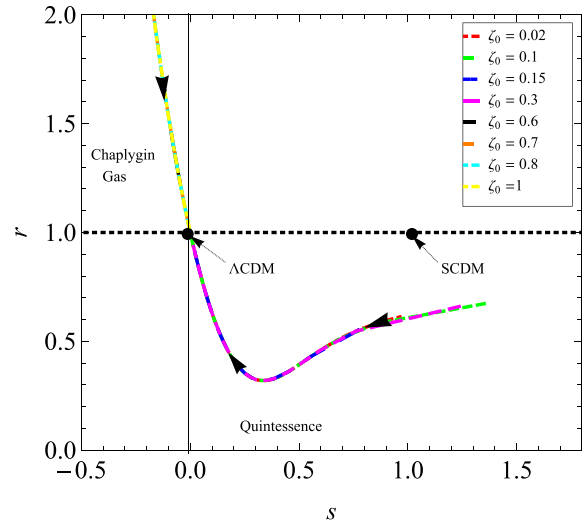

(a)

Fig. 3 a The $r-s$ trajectories are plotted in $r-s$ plane for different values of $\zeta_{0}>0$ with $\omega_{d}=-0.5, \alpha=0.8502$ and $\beta=0.4817$. The arrows represent the directions of the evolutions of statefinder diagnostic pair with time. The curves are coinciding with each other for smaller and larger values of $\zeta_{0}$. b The $r-q$ trajectories are plotted in $r-q$

In previous Sect. 2, we see that the diagnostic pair is constant in the absence of viscous term. As we can observe from the above two equations that in the limit of $\left(t-t_{0}\right) \rightarrow \infty$, the model approaches to $\{r, s\} \rightarrow\{1,0\}$ and for this limit we get $q \rightarrow-1$. We draw the trajectories of the statefinder pair $\{r, s\}$ in $r-s$ plane for different values of constant $\zeta_{0}$ with $\omega_{d}=-0.5, H_{0}=t_{0}=1, \alpha=0.8502$ and $\beta=0.4817$ as shown in Fig. 3a. Here, we observe that the model approaches to $\{r, s\} \rightarrow\{1,0\}$ for all positive values of $\zeta_{0}$. In Fig. 3a, the fixed points $\{r, s\}=\{1,1\}$ and $\{r, s\}=\{1,0\}$ are shown as $S C D M$ and $\Lambda C D M$ models, respectively.

It is observed from figures that the statefinder diagnostic of our model can discriminate from other DE models. For example, in quiessence with constant EoS parameter [37,38] and the Ricci dark energy (RDE) model [66], the trajectory in $r-s$ plane is a vertical segment, i.e. $s$ is constant during the evolution of the universe whereas the trajectories for the Chaplygin gas $(C G)$ [67] and the quintessence (inverse power-law) models $(Q)[37,38]$ are similar to arcs of a parabola (downward and upward) lying in the regions $s<0, r>1$ and $s>0, r<1$, respectively. In modified NHDE model [68], the trajectory in $r-s$ is from left to right. In holographic dark energy model with future event horizon $[69,70]$ its evolution starts from the point $s=2 / 3, r=1$ and ends at $\Lambda C D M$ model fixed point in future.

In Fig. 3a, the plot reveals that the $r-s$ plane can be divided into two regions $r<1, s>0$ and $r>1, s<0$ which are showing the similar characteristics to $Q$ and $C G$ models, respectively. The present model starts in both regions $r<1, s>0$ and $r>1, s<0$, and end on the $\Lambda C D M$ point in the $r-s$ plane in far future. The trajectories in the right side of the vertical line correspond to the different values of

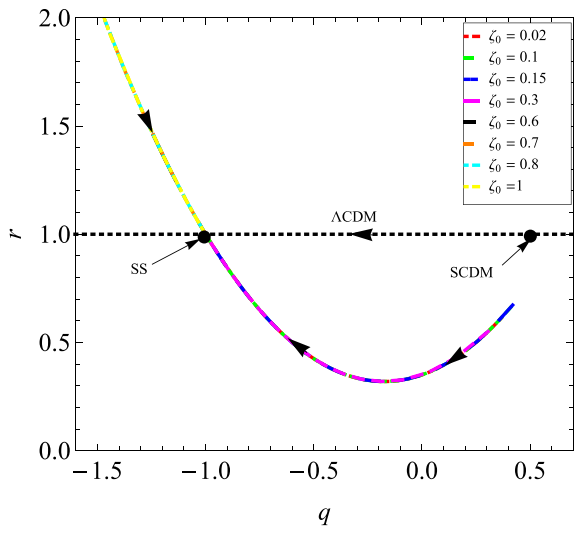

(b)

plane for different values of $\zeta_{0}>0$ with $\omega_{d}=-0.5, \alpha=0.8502$ and $\beta=0.4817$. The arrows represent the directions of the time evolution pair $\{r, q\}$ with time. The curves are coinciding with each other for smaller and larger values of $\zeta_{0}$

$\zeta_{0}$, i.e., $\zeta_{0}=0.02, \zeta_{0}=0.10, \zeta_{0}=0.15$ and $\zeta_{0}=0.30$ lying in the range $0<\zeta_{0} \leq 0.57$ whereas the trajectories to the left side of the vertical line correspond to $\zeta_{0}>0.57$, i.e., $\zeta_{0}=0.60, \zeta_{0}=0.70, \zeta_{0}=0.80$ and $\zeta_{0}=1.00$. This reveals that smaller values of $\zeta_{0}$ give the model similar to $Q$ model and larger values correspond to the $C G$ model. We find that the evolutions are coinciding each other for all different values of $\zeta_{0}$ in both regions.

We also study the evolutionary behaviour of constant viscous NHDE model in $r-q$ plane. For different values of $\zeta_{0}$, as taken in $\{r, s\}$, the trajectories are shown in Fig. $3 \mathrm{~b}$ for $w_{d}=-0.5, H_{0}=t_{0}=1, \alpha=0.8502$ and $\beta=0.4817$. The $S C D M$ model and steady state $(S S)$ model corresponds to fixed point $\{r, q\}=\{1,0.5\}$ and $\{r, q\}=\{1,-1\}$, respectively. It can be seen that there is a sign change of $q$ from positive to negative which explain the recent phase transition. The trajectories show that viscous NHDE models commence evolving from different points for different values of $\zeta_{0}$ with respect to $\Lambda C D M$ which starts from $S C D M$ fixed point. The viscous NHDE model always converges to $S S$ model as $\Lambda C D M, Q$ and $C G$ models in late-time evolution of the universe. Thus, the constant viscous NHDE model is compatible with $Q$ and $C G$ models.

The above discussion concludes the effect of viscous term in NHDE model. Let us discuss the model in view point of model parameters $\alpha$ and $\beta$. Figure $4 \mathrm{a}, \mathrm{b}$ show the trajectories in $r-s$ and $r-q$ planes, respectively, for the different values of $\alpha$ and $\beta$ with constant $\omega_{d}=-0.5, H_{0}=t_{0}=1$ and $\zeta_{0}=0.02$. The arrows in the diagram denote the evolution directions of the statefinder trajectories and $r-q$ trajectories. From Fig. 4a, we observe that for this fixed value of $\zeta_{0}$ the constant viscous NHDE model always correspond to the $Q$ 
Fig. 4 a The $r-s$ trajectories are plotted in $r-s$ plane for different values of $\alpha$ and $\beta$ with $\omega_{d}=-0.5$ and $\zeta_{0}=0.02$. The arrows represent the directions of the evolutions of statefinder diagnostic pair with time. $\mathbf{b}$ The $r-q$ trajectories are plotted in $r-q$ plane for different values of $\alpha$ and $\beta$ with $\omega_{d}=-0.5$ and $\zeta_{0}=0.02$. The arrows represent the directions of the time evolution pair $\{r, q\}$ with time

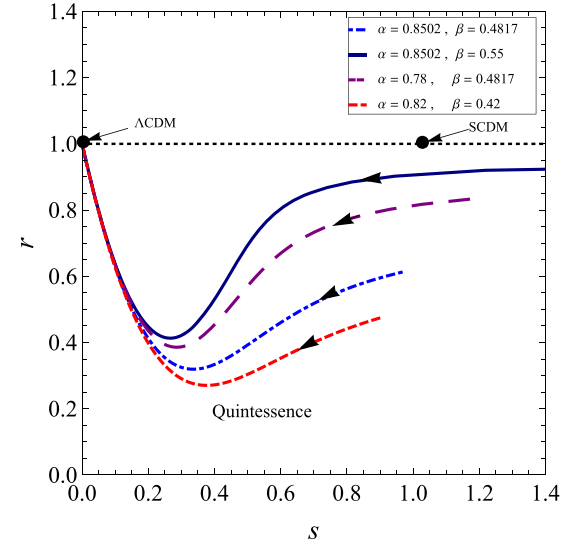

(a)

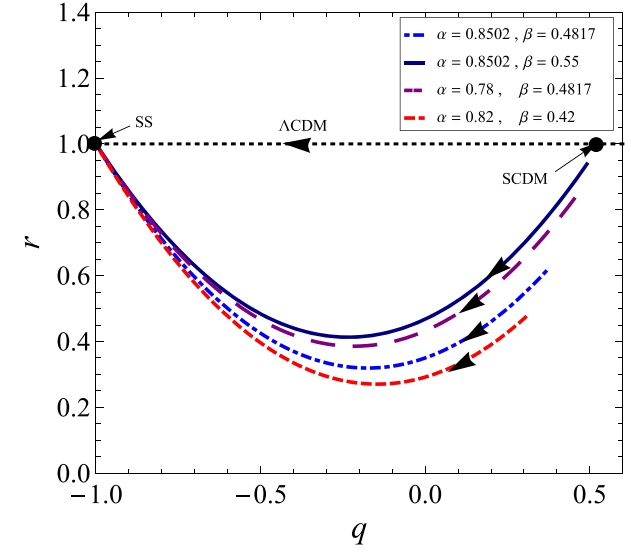

(b) model. It may start from the vicinity of $S C D M$ model in early time of evolution for some values of $\alpha$ and $\beta$, e.g., $(\alpha, \beta)=(0.8502,0.55)$. In late-time of evolution the model always converges to $\Lambda C D M$ model for any values of $(\alpha, \beta)$.

The panel (b) of Fig. 4 shows the time evolution of the $r-q$ trajectories in $r-q$ plane. The horizontal line at $r=1$ corresponds to the time evolution of the $\Lambda C D M$ model. The signature change from positive to negative in $q$ clearly explain the phase transition of the universe. The constant viscous NHDE model may start from the vicinity of the SCDM model $(\{r, q\}=\{1,0.5\})$ for some values of $\alpha$ and $\beta$ (e.g., $\alpha=0.8502, \beta=0.55$ ). However, the constant viscous NHDE model approaches to the $S S$ model as the $\triangle C D M$ and $Q$ models in future. Thus, the viscous NHDE model is compatible with the $\triangle C D M$ and $Q$ models with variables model parameters and constant value of $\zeta_{0}$.

Thus, we conclude that our model corresponds to both $Q$ and $C G$ models for the different values of viscous coefficient $\zeta_{0}$ whereas for the different values of model parameters $\alpha$ and $\beta$ with respect to the fixed value of $\zeta_{0}$, our model only corresponds to $Q$ model. Hence, we can conclude that due to the viscosity NHDE model is compatible with the $Q$ and $C G$ models. By above analysis, we can say that the bulk viscous coefficient and model parameters play the important roles in the evolution of the universe, i.e., they both determine the evolutionary behavior as well as the ultimate fate of the universe.

\section{Om diagnostic}

In addition to statefinder $\{r, s\}$, another diagnostic model, $\operatorname{Om}(z)$ is widely used to discriminate DE models. It is a new geometrical diagnostic which combines Hubble parameter $H$ and redshift $z$. The $O m(z)$ diagnostic [39] has been proposed to differentiate $\Lambda C D M$ to other DE models. Many authors [71-73] have studied the DE models based on $\mathrm{Om}(z)$ diagnostic. Its constant behaviour with respect to $z$ represents that $\mathrm{DE}$ is a cosmological constant $(\Lambda C D M)$. The positive slope of $O m(z)$ with respect to $z$ shows that the DE behaves as phantom and negative slope shows that DE behaves like quintessence. According to Ref. [39], Om(z) parameter for spatially flat universe is defined as

$\operatorname{Om}(z)=\frac{\frac{H^{2}(z)}{H_{0}^{2}}-1}{(1+z)^{3}-1}$,

where $H_{0}$ is the present value of the Hubble parameter. Since the $O m(z)$ involves only the first derivative of scale factor, so it is easier to reconstruct it as compare to statefinder parameters. It has been shown that the slope of $\operatorname{Om}(z)$ can distinguish dynamical dark energy from the cosmological constant in a robust manner, both with and without reference to the value of the matter density.

On substituting the required value of $H(z)$ from (22) in (32), we get the value of $\operatorname{Om}(z)$ as

$O m(z)=\frac{\left[\frac{\zeta_{0}}{H_{0}}+\left\{1+\alpha \omega_{d}-\frac{\zeta_{0}}{H_{0}}\right\}(1+z)^{\frac{3\left(1+\alpha \omega_{d}\right)}{2+3 \beta \omega_{d}}}\right]^{2}-\left(1+\alpha \omega_{d}\right)^{2}}{\left(1+\alpha \omega_{d}\right)^{2}\left[(1+z)^{3}-1\right]}$.

For comparison, we plot $\operatorname{Om}(z)$ trajectory with respect to $z$ for different values of $\zeta_{0}>0$ (or $\alpha$ and $\beta$ ) with fixed $\alpha$ and $\beta$ (or $\zeta_{0}$ ), with $H_{0}=1$ and $\omega_{d}=-0.5$ as shown in Fig. 5. From Fig. 5a, we observe that for $0<\zeta_{0} \leq 0.57$, the trajectory shows the negative slope, i.e., the DE behaves like quintessence and for $\zeta_{0}>0.57$, the positive slope of the Om trajectory is observed, i.e., the DE behaves as phantom. For the late future stage of evolution when $z=-1$, we get $\operatorname{Om}(z)=1-\frac{\zeta_{0}^{2}}{H_{0}^{2}\left(1+\alpha \omega_{d}\right)^{2}}$, which is the constant value of $\operatorname{Om}(z)$. Thus for $z=-1$, the DE will correspond to $\Lambda C D M$.

The Fig. 5 b shows the $\operatorname{Om}(z)$ trajectory for different values of model parameters $\alpha$ and $\beta$ with fixed $\zeta_{0}=0.02, \omega_{d}=$ -0.5 and $H_{0}=1$. This trajectory only shows the negative curvature which imply that the DE behaves like quintessence. 
Fig. 5 a The $\operatorname{Om}(z)$

evolutionary diagram of viscous NHDE for different values of $\zeta_{0}>0$ with fixed $\omega_{d}=-0.5$, $\alpha=0.8502$ and $\beta=0.4817$. b The $\operatorname{Om}(z)$ evolutionary diagram of viscous NHDE for different values of $\alpha$ and $\beta$ with fixed $\zeta_{0}=0.02$ with $\omega_{d}=-0.5$

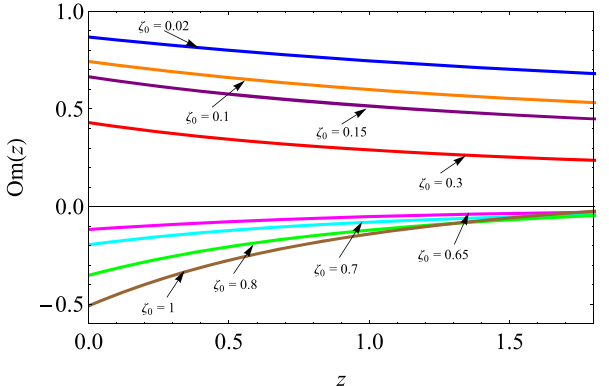

(a)

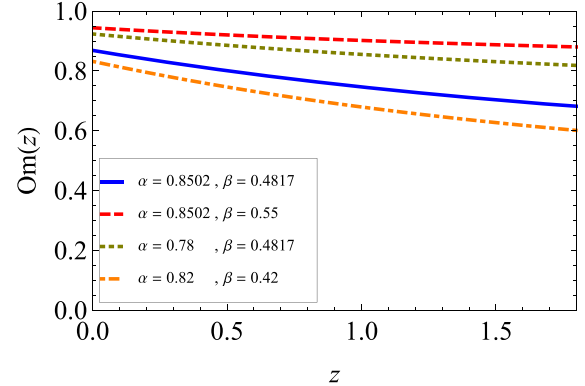

(b)
From the above discussion with constant bulk viscous coefficient, we find that the constant $\zeta_{0}$ ( or cosmological parameters $\alpha$ and $\beta$ ) play important roles in the evolution of the universe, i.e., they both determine the evolutionary behavior as well as the ultimate fate of the universe.

\subsection{Solution for variable bulk viscosity coefficient}

In this section, we consider two cases: (i) $\zeta_{0}=0$ and $\zeta_{1} \neq 0$, and (ii) $\zeta_{0} \neq 0$ and $\zeta_{1} \neq 0$.

Case (i) $\zeta_{0}=\mathbf{0}$ and $\zeta_{1} \neq \mathbf{0}$ :

In this case, the bulk viscosity coefficient given in (15) reduces to

$\zeta=\zeta_{1} H$

which shows that the bulk viscous coefficient is directly proportional to Hubble parameter.

Using (34) into (16), we get

$\dot{H}+\frac{3\left(1-\zeta_{1}+\alpha \omega_{d}\right)}{\left(2+3 \beta \omega_{d}\right)} H^{2}=0$.

The above equation is similar to the Eq.(5) obtained in the case of non-viscous NHDE model in Sect. 2. The solution of (35) for $H$ in terms of $t$ is given by

$H=\frac{1}{c_{3}+\frac{3\left(1-\zeta 1+\alpha \omega_{d}\right)}{\left(2+3 \beta \omega_{d}\right)} t}$

where $c_{3}$ represents the constant of integration. The scale factor can be obtained as

$$
\begin{gathered}
a=a_{0}\left[1+\frac{3\left(1-\zeta_{1}+\alpha \omega_{d}\right) H_{0}}{\left(2+3 \beta \omega_{d}\right)}\left(t-t_{0}\right)\right]^{\frac{\left(2+3 \beta \omega_{d}\right)}{3\left(1-\zeta_{1}+\alpha \omega_{d}\right)}}, \\
\qquad \text { for } \zeta_{1} \neq\left(1+\alpha \omega_{d}\right), \quad \beta \neq-\frac{2}{3 \omega_{d}}
\end{gathered}
$$

The scale factor varies as power-law expansion. Now, the DP is

$q=\frac{3\left(1-\zeta_{1}+\alpha \omega_{d}\right)}{\left(2+3 \beta \omega_{d}\right)}-1$.

which is a constant value. Such form of $\zeta$ gives no transition phase. The positive or negative sign of $q$ depends on whether
$3 \zeta_{1}<\left(1+3(\alpha-\beta) \omega_{d}\right)$ or $3 \zeta_{1}>\left(1+3(\alpha-\beta) \omega_{d}\right)$, respectively.

Now, the statefinder parameter can be given as

$r=1-\frac{9\left(1+\alpha \omega_{d}-\zeta_{1}\right)}{\left(2+3 \beta \omega_{d}\right)}+\frac{18\left(1+\alpha \omega_{d}-\zeta_{1}\right)^{2}}{\left(2+3 \beta \omega_{d}\right)^{2}}$,

and

$s=\frac{2\left(1+\alpha \omega_{d}-\zeta_{1}\right)}{\left(2+3 \beta \omega_{d}\right)}$.

In this case the statefinder pair is constant. In the limit of $\zeta_{1} \rightarrow\left(1+\alpha \omega_{d}\right)$, the statefinder pair $\{r, s\} \rightarrow\{1,0\}$ and for $\zeta_{1}=\frac{(2 \alpha-3 \beta) \omega_{d}}{2}$, this model behaves as SCDM, i.e., $\{r, s\}=$ $\{1,1\}$.

Case (ii) $\zeta_{0} \neq \mathbf{0}$ and $\zeta_{1} \neq 0$ :

Let us consider the more general form of the bulk viscous coefficient, i.e., $\zeta=\zeta_{0}+\zeta_{1} H$. Using (15) into (16), we get

$\dot{H}+\frac{3\left(1-\zeta_{1}+\alpha \omega_{d}\right)}{\left(2+3 \beta \omega_{d}\right)} H^{2}-\frac{3 \zeta_{0}}{\left(2+3 \beta \omega_{d}\right)} H=0$.

Solving the above equation, we get the Hubble parameter in terms of $t$ as

$$
\begin{aligned}
H= & H_{0} e^{\frac{3 \zeta_{0}\left(t-t_{0}\right)}{\left(2+3 \beta \omega_{d}\right)}} \\
& \times\left[1+\frac{H_{0}\left(1-\zeta_{1}+\alpha \omega_{d}\right)}{\zeta_{0}}\left\{e^{\frac{3 \zeta_{0}\left(t-t_{0}\right)}{\left(2+3 \beta \omega_{d}\right)}}-1\right\}\right]^{-1},
\end{aligned}
$$

where $H_{0}$ is the present value of the Hubble parameter and we have made the assumption that the present value of scale factor is $a_{0}=1$. The solution of (42) for the scale factor $a$ in terms of $t$ is given by

$a=\left[1+\frac{H_{0}\left(1-\zeta_{1}+\alpha \omega_{d}\right)}{\zeta_{0}}\left\{e^{\frac{3 \zeta_{0}\left(t-t_{0}\right)}{\left(2+3 \beta \omega_{d}\right)}}-1\right\}\right]^{\frac{\left(2+3 \beta \omega_{d}\right)}{3\left(1-\zeta_{1}+\alpha \omega_{d}\right)}}$,
$\quad$ for $\zeta_{0} \neq 0, \quad \zeta_{1} \neq\left(1+\alpha \omega_{d}\right)$

Here, we get an exponential type scale factor with the viscous terms. As $\left(t-t_{0}\right) \rightarrow 0$, the scale factor behaves as

$a \rightarrow\left[1+\frac{3 H_{0}\left(1-\zeta_{1}+\alpha \omega_{d}\right)\left(t-t_{0}\right)}{\left(2+3 \beta \omega_{d}\right)}\right]^{\frac{\left(2+3 \beta \omega_{d}\right)}{3\left(1-\zeta_{1}+\alpha \omega_{d}\right)}}$, 
which shows power-law expansion in early time. On the other hand, if $\zeta_{0}=H_{0}\left(1-\zeta_{1}+\alpha \omega_{d}\right)$ or $\left(t-t_{0}\right) \rightarrow \infty$, we obtain

$a(t)=\exp \left(\frac{3 \zeta_{0}\left(t-t_{0}\right)}{\left(2+3 \beta \omega_{d}\right)}\right)$.

This case corresponds the de Sitter universe which shows accelerated expansion in the later time of evolution.

Now, with the help of (43), the Hubble parameter in terms of scale factor can be written as

$$
\begin{aligned}
H(a)= & \frac{H_{0}}{\left(1-\zeta_{1}+\alpha \omega_{d}\right)} \\
& \times\left[\frac{\zeta_{0}}{H_{0}}+\left\{\left(1-\zeta_{1}+\alpha \omega_{d}\right)-\frac{\zeta_{0}}{H_{0}}\right\} a^{-\frac{3\left(1-\zeta_{1}+\alpha \omega_{d}\right)}{\left(2+3 \beta \omega_{d}\right)}}\right] .
\end{aligned}
$$

This equations shows that if both $\zeta_{0}$ and $\zeta_{1}$ are zero, the Hubble parameter, $H=H_{0} a^{\frac{-3\left(1+\alpha \omega_{d}\right)}{\left(2+3 \alpha \omega_{d}\right)}}$, which corresponds to non-viscous NHDE model. When $\zeta_{1}=0, H$ reduces to Eq. (22) which is the case of constant viscosity.

The derivative of $\dot{a}$ with respect to $a$ can be obtained from (46), which is given by

$$
\begin{aligned}
\frac{d \dot{a}}{d a}= & \frac{H_{0}}{\left(1-\zeta_{1}+\alpha w_{d}\right)}\left[\frac{\zeta_{0}}{H_{0}}-\left\{\left(1-\zeta_{1}+\alpha w_{d}\right)-\frac{\zeta_{0}}{H_{0}}\right\}\right. \\
& \left.\times\left(\frac{\left(1+3(\alpha-\beta) w_{d}-3 \zeta_{1}\right)}{2+3 \beta w_{d}}\right) a^{-\frac{3\left(1-\zeta_{1}+\alpha w_{d}\right)}{\left(2+3 \beta w_{d}\right)}}\right] .
\end{aligned}
$$

Equating (47) to zero to get the transition scale factor $a_{T}$ as

$a_{T}=\left[\frac{\left(1+3(\alpha-\beta) w_{d}-3 \zeta_{1}\right)\left\{\left(1-\zeta_{1}+\alpha w_{d}\right) H_{0}-\zeta_{0}\right\}}{\left(2+3 \beta w_{d}\right) \zeta_{0}}\right]^{\frac{\left(2+3 \beta w_{d}\right)}{3\left(1-\zeta_{1}+\alpha w_{d}\right)}}$

The corresponding transition redshift $z_{T}$ is

$z_{T}=\left[\frac{\left(1+(\alpha-\beta) w_{d}-3 \zeta_{1}\right)\left\{\left(1-\zeta_{1}+\alpha w_{d}\right) H_{0}-\zeta_{0}\right\}}{\left(2+3 \beta w_{d}\right) \zeta_{0}}\right]^{-\frac{\left(2+3 \beta w_{d}\right)}{3\left(1-\zeta_{1}+\alpha w_{d}\right)}}-1$.

It can be observed that for $\left(\zeta_{0}+\zeta_{1} H_{0}\right)=\frac{\left\{1+3(\alpha-\beta) w_{d}\right\} H_{0}}{3}$, the transition from decelerated phase to accelerated phase occurs at $a_{T}=1$ or $z_{T}=0$, which corresponds to the present time of the universe. By considering the observational value $\alpha=0.8502$ and $\beta=0.4817$ along with $\omega_{d}=-0.5, H_{0}=1$, we get $\left(\zeta_{0}+\zeta_{1}\right)=0.15$. A plot of the evolution of the scale factor is given in Fig. 6. Thus, for $0<\left(\zeta_{0}+\zeta_{1}\right) \leq 0.15$ the scale factor has earlier deceleration phase followed by an acceleration phase in later stage of the evolution. The transition from the decelerated to accelerated expansion depends on the viscosity $\zeta_{0}$ and $\zeta_{1}$ as shown above. For $\left(\zeta_{0}+\zeta_{1}\right)>0.15$, the transition takes place in past of the universe and the scale factor increases with accelerated rate forever.

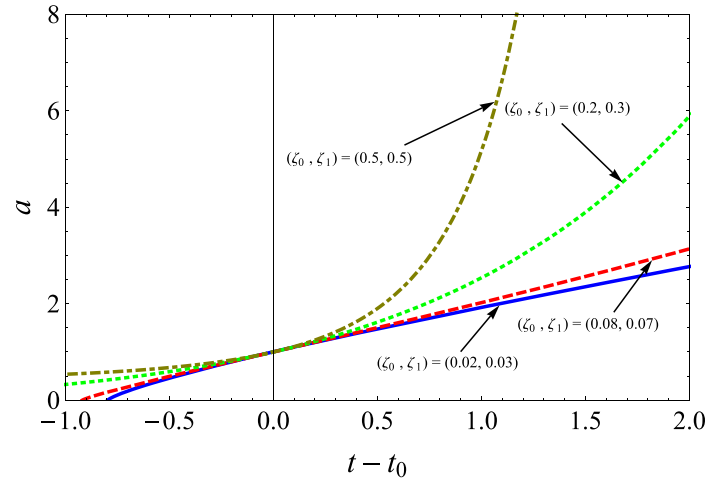

Fig. 6 The plot of the scale factor with respect to $t-t_{0}$ for different values of $\zeta_{0}, \zeta_{1}$ when $\zeta_{0}>0$ and $\zeta_{1}>0$ with $\omega_{d}=-0.5, \alpha=0.8502$ and $\beta=0.4817$

The transition may also be discussed through the evolution of DP. In this case, we get

$q=\frac{3\left\{\left(1-\zeta_{1}+\alpha \omega_{d}\right)-\frac{\zeta_{0}}{H_{0}}\right\}}{\left(2+3 \beta \omega_{d}\right)} e^{-\frac{3 \zeta_{0}\left(t-t_{0}\right)}{\left(2+3 \beta \omega_{d}\right)}}-1$,

which is a time-dependent value of DP, which may describe the transition phase of the universe. It can be observed that DP must change its sign at $t=t_{0}$. This time can be achieved if $3\left(\zeta_{0}+\zeta_{1} H_{0}\right)=\left\{1+3(\alpha-\beta) w_{d}\right\} H_{0}$. The sign of $q$ is positive for $t<t_{0}$ and it is negative for $t>t_{0}$. The values of $\zeta_{0}$ and $\zeta_{1}$ can be obtained for a given values of $\omega_{d}, \alpha$ and $\beta$, which may be obtained from observation, or vice-versa.

From (50), DP can be written in terms of scale factor as

$$
\begin{aligned}
q(a)= & \frac{\left\{3\left(1-\zeta_{1}+\alpha \omega_{d}\right)-3 \zeta_{0}\right\}}{\left(2+3 \beta \omega_{d}\right)} \\
& \times\left[\frac{\left(1-\zeta_{1}+\alpha \omega_{d}\right)}{\left(a^{\frac{3\left(1-\zeta_{1}+\alpha \omega_{d}\right)}{2+3 \beta \omega_{d}}}-1\right) \zeta_{0}+\left(1-\zeta_{1}+\alpha \omega_{d}\right)}\right]-1 .
\end{aligned}
$$

In terms of red shift $z$, the above equation becomes

$$
\begin{aligned}
q(z)= & \frac{\left\{3\left(1-\zeta_{1}+\alpha \omega_{d}\right)-3 \zeta_{0}\right\}}{\left(2+3 \beta \omega_{d}\right)} \\
& \times\left[\frac{\left(1-\zeta_{1}+\alpha \omega_{d}\right)}{\left((1+z)-\frac{3\left(1-\zeta_{1}+\alpha \omega_{d}\right)}{2+3 \beta \omega_{d}}-1\right) \zeta_{0}+\left(1-\zeta_{1}+\alpha \omega_{d}\right)}\right]-1 .
\end{aligned}
$$

When the bulk viscous parameter and all other parameter are zero, the deceleration parameter $q=1 / 2$, which corresponds to a decelerating matter dominated universe with null bulk viscosity. However, when only the bulk viscous term $\zeta_{0}=0$ and $\zeta_{1} \neq 0$, the value of $q$ is same as obtained in Eq. (38) for 
case (i) of variable viscous NHDE model, and when $\zeta_{0} \neq 0$ and $\zeta_{1}=0$, Eq. (51) reduces to Eq. (27) of constant viscous coefficient.

The present value of $q$ corresponds to $z=0$ or $a=1$ is,

$q_{0}=q(a=1)=\frac{3\left(1-\zeta_{1}+\alpha \omega_{d}\right)-3 \zeta_{0}}{\left(2+3 \beta \omega_{d}\right)}-1$.

This equation shows that if $3\left(\zeta_{0}+\zeta_{1}\right)=\left[1+3(\alpha-\beta) \omega_{d}\right]$, the deceleration parameter $q=0$. This implies that the transition into the accelerating phase would occur at the present time. The current DP $q_{0}<0$ if $3\left(\zeta_{0}+\zeta_{1}\right)>\left[1+3(\alpha-\beta) \omega_{d}\right]$, implying that the present universe is in the accelerating epoch and it entered this epoch at an early stage. But $q_{0}>0$ if $3\left(\zeta_{0}+\zeta_{1}\right)<\left[1+3(\alpha-\beta) \omega_{d}\right]$ implying that the present universe is decelerating and it will be entering the accelerating phase at a future time. For the observational value $\alpha=0.8502$ and $\beta=0.4817$ with $\omega_{d}=-0.5$ and $H_{0}=1$, we get $\left(\zeta_{0}+\zeta_{1}\right)=0.15$ which gives $q_{0}=0$. Thus for this

Statefinder diagnostic

As we have mentioned above, the scale factor and deceleration parameter have been discussed to explain the accelerating universe with viscous term or model parameters. So it is necessary to distinguished these models in a modelindependent manner. In what follows we will apply two geometrical approaches to viscous NHDE model, i.e., the statefinder and $\mathrm{Om}$ diagnostic from which we can compute the evolutionary trajectories with ones of the $\Lambda C D M$ model to show the difference among them.

In this case, the statefinder parameters defined in Eq. (10) can be evaluated as

$$
\begin{aligned}
r= & +\frac{9\left(\frac{\zeta_{0}}{H_{0}}-\left(1-\zeta_{1}+\alpha \omega_{d}\right)\right)\left(1-\frac{\left(1-\zeta_{1}+\alpha \omega_{d}\right)}{\left(2+3 \beta \omega_{d}\right)}\right) e^{-\frac{3 \zeta_{0}\left(t-t_{0}\right)}{\left(2+3 \beta \omega_{d}\right)}}}{\left(2+3 \beta \omega_{d}\right)} \\
& +\frac{9\left(\frac{\zeta_{0}}{H_{0}}-\left(1-\zeta_{1}+\alpha \omega_{d}\right)\right)^{2} e^{-\frac{6 \zeta_{0}\left(t-t_{0}\right)}{\left(2+3 \beta \omega_{d}\right)}}}{\left(2+3 \beta \omega_{d}\right)^{2}}
\end{aligned}
$$

and

$s=\frac{\frac{2\left(\frac{\zeta_{0}}{H_{0}}-\left(1-\zeta_{1}+\alpha \omega_{d}\right)\right)\left(1-\frac{1-\zeta_{1}+\alpha \omega_{d}}{\left(2+3 \beta \omega_{d}\right)}\right)}{\left(2+3 \beta \omega_{d}\right)} e^{-\frac{3 \zeta_{0}\left(t-t_{0}\right)}{\left(2+3 \beta \omega_{d}\right)}}+\frac{2\left(\frac{\zeta_{0}}{H_{0}}-\left(1-\zeta_{1}+\alpha \omega_{d}\right)\right)^{2}}{\left(2+3 \beta \omega_{d}\right)^{2}} e^{-\frac{6 \zeta_{0}\left(t-t_{0}\right)}{\left(2+3 \beta \omega_{d}\right)}}}{\frac{2\left(\left(1-\zeta_{1}+\alpha \omega_{d}\right)-\frac{\zeta_{0}}{H_{0}}\right)}{\left(2+3 \beta \omega_{d}\right)} e^{-\frac{3 \zeta_{0}\left(t-t_{0}\right)}{\left(2+3 \beta \omega_{d}\right)}}-1}$.

value set, the transition into accelerating phase would occur at present time. If $\left(\zeta_{0}+\zeta_{1}\right)>0.15, q_{0}<0$, i.e., the universe is in accelerating phase and it entered this epoch at an early stage. If $\left(\zeta_{0}+\zeta_{1}\right)<0.15, q_{0}>0$, i.e., the universe is in decelerating epoch and it will be entered into the accelerated phase in future. This result is verified graphically which is represented by Fig. 7a. The Fig. 7b shows the $q-a$ graph in $q-a$ plane to discuss the evolution of the universe with respect to model parameters $\alpha$ and $\beta$. Here, the signature change in the value of DP can be seen by the figure. From above discussion we say that both viscous coefficient and model parameter have their own role in the evolution of the universe. Some values of bulk viscous term gives the accelerated phase from the beginning and continues to be accelerated in late time.
From (54) and (55) it can be observed that the viscous NHDE model converges to $\{r, s\} \rightarrow\{1,0\}$ in the limit of $\left(t-t_{0}\right) \rightarrow \infty$. This can also be achieved at $\left(\zeta_{0}+H_{0} \zeta_{1}\right)=$ $H_{0}\left(1+\alpha \omega_{d}\right)$ but this is a very fixed point. Thus, the statefinder diagnostic fails to discriminate between $\triangle C D M$ and the NHDE model. Here, we obtain time-dependent statefinder pair which needs to study the general behavior. Let us see the effect of viscosity coefficients $\zeta_{0}, \zeta_{1}$ and model parameters $\alpha, \beta$ for the general form of variable viscous NHDE model. Figure 8a shows the $r-s$ trajectory in $r-s$ plane for different values of $\zeta_{0}$ and $\zeta_{1}$ with $H_{0}=t_{0}=1, \alpha=0.8502$ and $\beta=0.4817$. The model behaviors to $Q$ models for $0<\left(\zeta_{0}+\zeta_{1}\right) \leq 0.57$ and $C G$ models for $\left(\zeta_{0}+\zeta_{1}\right)>0.57$. The trajectories in Q-model and CG-model both converge to the $\Lambda C D M$ model in late time of evolution.
Fig. 7 a The $q-a$ graph in $q-a$ plane for different values of $\zeta_{0}>0$ and $\zeta_{1}>0$ with $\omega_{d}=-0.5, \alpha=0.8502$ and $\beta=0.4817$. b The $q-a$ graph in $q-a$ plane for different values of $\alpha$ and $\beta$ with $\zeta_{0}=0.2$, $\zeta_{1}=0.3$ and $\omega_{d}=-0.5$

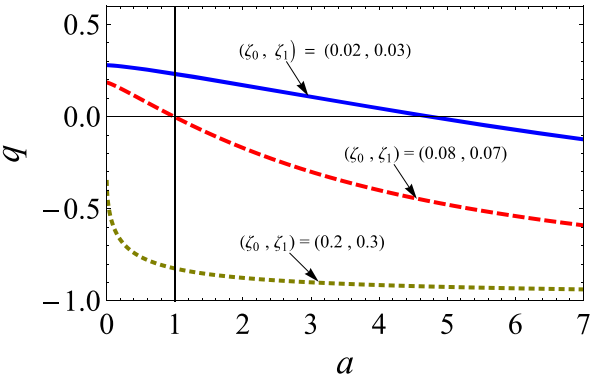

(a)

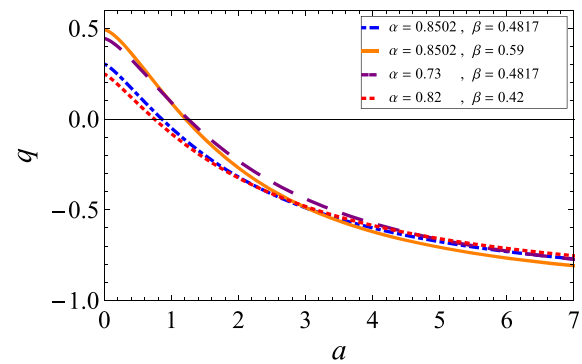

(b) 
Fig. 8 a The $r-s$ trajectories are plotted in $r-s$ plane for different values of $\zeta_{0}>0$ and $\zeta_{1}>0$ with $\omega_{d}=-0.5$, $\alpha=0.8502$ and $\beta=0.4817$. The arrows represent the directions of the evolutions of statefinder diagnostic pair with time. b The $r-q$ trajectories are plotted in $r-q$ plane for different values of $\zeta_{0}>0$ and $\zeta_{1}>0$ with $\omega_{d}=-0.5$, $\alpha=0.8502$ and $\beta=0.4817$. The arrows represent the directions of the time evolution pair $\{r, q\}$ with time

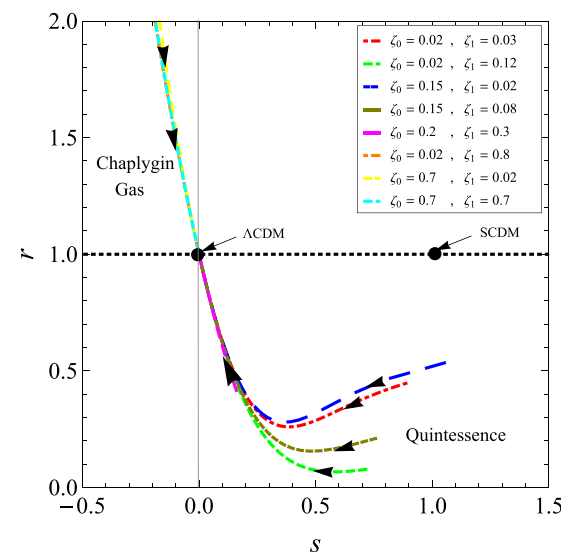

(a)

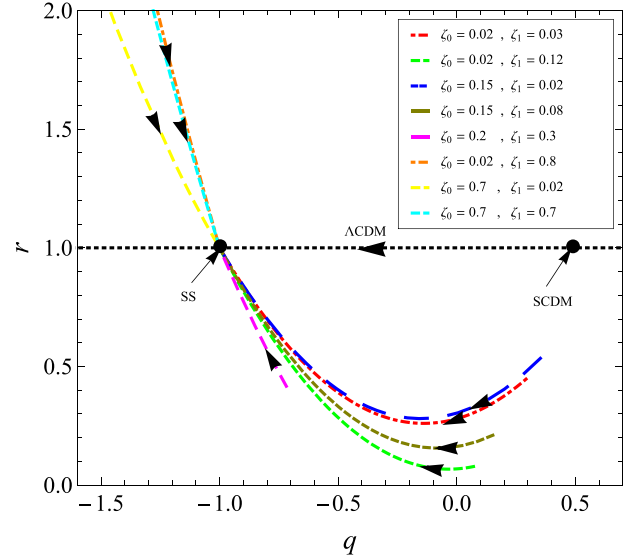

(b)
Figure $8 \mathrm{~b}$ shows the time evolution of $\{r, q\}$ pair in $r-q$ plane for different combinations of the values of $\zeta_{0}$ and $\zeta_{1}$ with $H_{0}=t_{0}=1, \alpha=0.8502$ and $\beta=0.4817$. The fixed points $\{r, q\}=\{1,0.5\}$ and $\{r, q\}=\{1,-1\}$ represents the $S C D M$ and $S S$ models, respectively. Since $q$ changes its sign from positive to negative with respect to time which shows the phase transition of the universe from deceleration to acceleration. In beginning this model behaves different from the $\Lambda C D M$ but in future it behaves same as $\triangle C D M$ which converges to $S S$ model in late-time. Hence the variable viscous NHDE model always converges to $S S$ model as $\Lambda C D M, Q$ and $C G$ models in late-time evolution of the universe. For all the ranges of $\left(\zeta_{0}+\zeta_{1}\right)$ the trajectories correspond to $Q$ and $C G$ models as in Fig. 8a. Thus the variable viscous NHDE model is compatible with both $Q$ and $C G$ models.

Thus, the viscosity coefficients are able to correspond to both $Q$ and $C G$ models for different combinations of $\zeta_{0}, \zeta_{1}$ and also explain the phase transition of the universe.

Now, we are curious to know the behaviour of variable viscous NHDE model with respect to the model parameters $\alpha$ and $\beta$. Here, Fig. 9a, b represents the $r-s$ and $r-q$ trajectories in $r-s$ and $r-q$ plane, respectively, for the different values of $\alpha$ and $\beta$ close to it's observational value with $\omega_{d}=-0.5, H_{0}=t_{0}=1, \zeta_{0}=0.02$ and $\zeta_{1}=0.03$. The evolutionary directions of both the trajectories are shown in the figures by the arrows. In Fig. 9a, we analysed that for this fixed value of $\zeta_{0}$ and $\zeta_{1}$ the $(r, s)$ trajectories are lying in the region corresponds to $r<1, s>0$ which shows that our model is similar to the $Q$ model. It also starts from the vicinity of SCDM model in early time of evolution for some values of $\alpha$ and $\beta$, e.g., $(\alpha, \beta)=(0.8502,0.59)$. It is different from RDE model and quiessence model as it produces the curved trajectories for any values of $(\alpha, \beta)$ close to observational value which approach to $\Lambda C D M$ in late-time of evolution as the $Q$ model tends to $\Lambda C D M$ model in late-time of evolution.

The $r-q$ trajectories in $r-q$ plane are shown by the Fig. 9b. This model is also able to explain the phase transition of the universe. It also starts from the neighbourhood of the SCDM model for some values of $\alpha$ and $\beta$ (e.g., $\alpha=0.8502, \beta=0.55$ ) and approaches to $S S$ model in latetime for any value of $\alpha$ and $\beta$ close to the observational value. In future the variable viscous NHDE model approaches to the $S S$ model same as the $\Lambda C D M$ and $Q$ models. Thus the viscous NHDE model is compatible with the $\Lambda C D M$ and $Q$ models.

Thus, we observed from Figs. 8 and 9 that viscous NHDE model is compatible to $Q$ and $C G$ models for different ranges of viscosity coefficients in the presence of the fixed observational value of model parameters whereas the model parameter in the presence of fixed value of viscosity coefficients approaches only to $Q$ model.

Om diagnostic

Let us discuss the another geometrical parameter, i.e., $\operatorname{Om}(z)$ diagnostic in viscous NHDE model. By substituting the required values in Eq. (32), we get the $\operatorname{Om}(z)$ diagnostic for $\zeta=\zeta_{0}+\zeta_{1} H$ as

$$
O m(z)=\frac{\left[\frac{\zeta_{0}}{H_{0}}+\left\{\left(1-\zeta_{1}+\alpha \omega_{d}\right)-\frac{\zeta_{0}}{H_{0}}\right\}(1+z)^{\frac{3\left(1-\zeta_{1}+\alpha \omega_{d}\right)}{2+3 \beta \omega_{d}}}\right]^{2}-\left(1-\zeta_{1}+\alpha \omega_{d}\right)^{2}}{\left(1-\zeta_{1}+\alpha \omega_{d}\right)^{2}\left[(1+z)^{3}-1\right]} .
$$


Fig. 9 a The $r-s$ trajectories are plotted in $r-s$ plane for different values of $\alpha$ and $\beta$ with $\omega_{d}=-0.5, \zeta_{0}=0.02$ and $\zeta_{1}=0.03$. The arrows represent the directions of the evolutions of statefinder diagnostic pair with time. b The $r-q$ trajectories are plotted in $r-q$ plane for different values of $\alpha$ and $\beta$ with $\omega_{d}=-0.5$, $\zeta_{0}=0.02$ and $\zeta_{1}=0.03$. The arrows represent the directions of the time evolution pair $\{r, q\}$ with time

Fig. 10 a The evolution of $\operatorname{Om}(z)$ versus the redshift $z$ for different values of $\zeta_{0}>0$ and $\zeta_{1}>0$ with $\omega_{d}=-0.5$, $\alpha=0.8502$ and $\beta=0.4817$. b The evolution of $\mathrm{Om}(z)$ versus the redshift $z$ for different values of $\alpha$ and $\beta$ with $\zeta_{0}=0.02$, $\zeta_{1}=0.03$ and $\omega_{d}=-0.5$

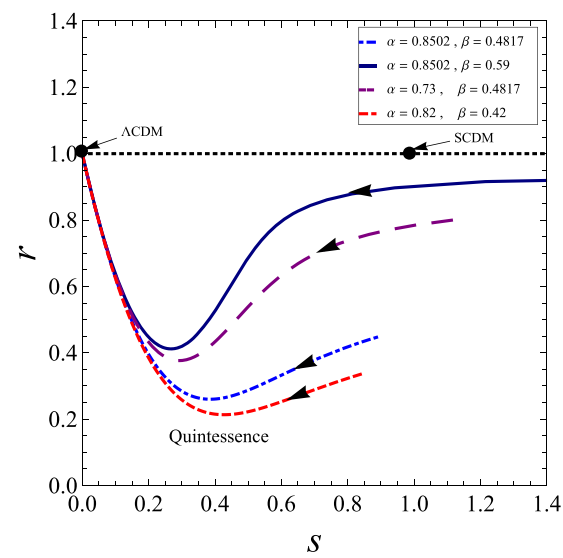

(a)

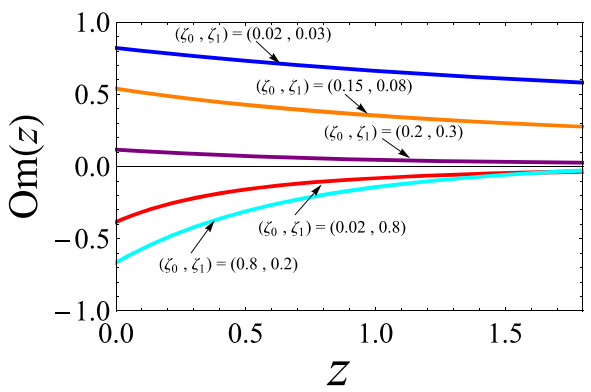

(a)

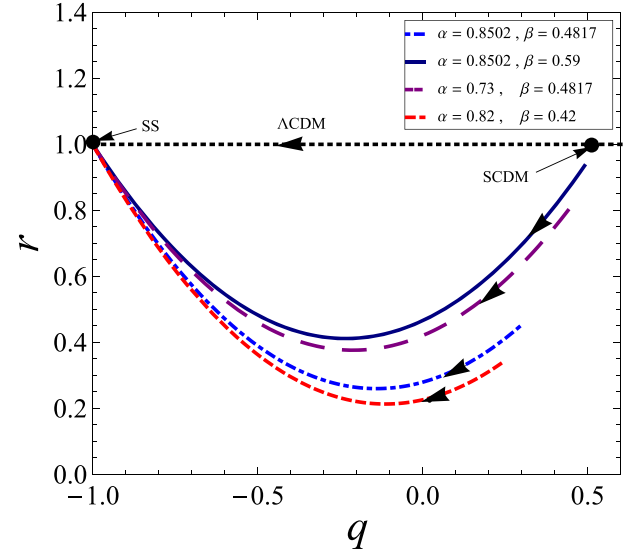

(b)

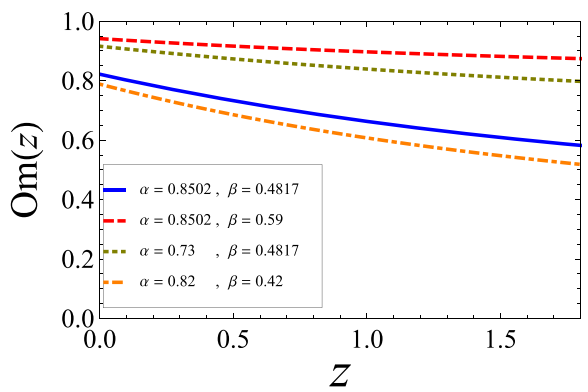

(b)
Figure 10a shows the $\operatorname{Om}(z)$ trajectory with respect to $z$ for different values of $\zeta_{0}>0$ and $\zeta_{1}>0$ corresponding to $\alpha=0.8502, \beta=0.4817, H_{0}=1$ and $\omega_{d}=-0.5$. Here, the trajectory represents the negative curvature, i.e., the viscous NHDE behaves as quintessence for the limit $0<\left(\zeta_{0}+\zeta_{1}\right) \leq$ 0.57 and it shows the positive curvature, i.e., the viscous DE behaves as phantom, for $\left(\zeta_{0}+\zeta_{1}\right)>0.57$ whereas for $z=$ -1 , i.e., in future time we get $\operatorname{Om}(z)=1-\frac{\zeta_{0}^{2}}{H_{0}^{2}\left(1-\zeta_{1}+\alpha \omega_{d}\right)^{2}}$, which is the constant value of $\operatorname{Om}(z)$. Thus for $z=-1$, the viscous NHDE will correspond to $\Lambda C D M$.

Figure 10b plot the $\operatorname{Om}(z)$ versus $z$ for different model parameters $\alpha$ and $\beta$ correspond to fixed $\zeta_{0}$ and $\zeta_{1}$. The graph shows that there is always negative curvature for any values of model parameters. This shows that the model behaviors similar to quintessence model.

\section{Conclusion}

We have studied some viscous cosmological NHDE models on the evolution of the universe, where the IR cutoff is given by the modified Ricci scalar, proposed by Granda and Olivers [29,30]. It has been tried to demonstrate that the bulk viscosity can also play the role as a possible candidate of DE. We have performed a detailed study of both non-viscous and viscous NHDE models. The component of this model is $\mathrm{DE}$ and pressureless DM. We have obtained the solutions for scale factor and deceleration parameter. We have also studied these models from two independent geometrical point of view, namely the statefinder parameter and $\mathrm{Om}$ diagnostic. We have studied the different possible scenarios of viscous NHDE and analyzed the evolution of the universe according to the assumption of bulk viscous coefficient $\zeta$. In the following we summarize the results obtained in different sections for non-viscous and viscous NHDE models.

In Sect. 2, we have investigated non-viscous NHDE in flat FRW universe. We have calculated the relevant cosmological parameters and their evolution. The evolution of scale factor has been studied. We have obtained power-law form of scale factor for which the model may decelerate or accelerate depending on the constraint of model parameters. The deceleration parameter is constant in this case. Therefore, the model can not describe the transition phase of the universe. The statefinder parameters are also constant. We have observed that the observed set of data of model parameters do not favor the NHDE model over the $\triangle \mathrm{CDM}$ as well as SC D M model. However, NHDE model behaves like SC DM model for $\alpha \rightarrow 3 \beta / 2$. It has been observed that this model approaches to $\{r, s\} \rightarrow\{1,0\}$ in the limit of $\alpha \rightarrow-1 / \omega_{d}$ but there is no such value of parameters which would clearly show the $\Lambda \mathrm{CDM}$.

In viscous NHDE model as discussed in Sect. 3, we have considered that the matter consists of viscous holographic dark energy and pressureless DM. We have assumed a most 
general form $\zeta=\zeta_{0}+\zeta_{1} H$ to observe the effect of bulk viscous coefficient in the evolution of the universe during early and late time. We have studied three cases: $\left(\zeta_{0} \neq 0, \zeta_{1}=0\right)$; $\left(\zeta_{0}=0, \zeta_{1} \neq 0\right)$ and $\left(\zeta_{0} \neq 0, \zeta_{1} \neq 0\right)$.

In the first case where we have constant bulk viscous coefficient, i.e., $\zeta=\zeta_{0}$, an exponential form of the scale factor is obtained. Therefore, the universe starts from a finite volume followed by an early decelerated phase and then transits into an accelerated phase in late time of evolution. As $\left(t-t_{0}\right) \rightarrow 0$, the scale factor reduces to the power-law form which corresponds to an early decelerated expansion. As $\left(t-t_{0}\right) \rightarrow \infty$, the scale factor tends to the exponential form which corresponds to acceleration similar to the de Sitter phase. The scale factor and red shift corresponding to the transition from decelerated to accelerated expansion has been obtained. The evolution of scale factor has been shown is Fig. 1. It is clear from Fig. 1 that if $\zeta_{0}=0.15$, transition from decelerated phase to accelerated phase occurs at $a_{T}=1$ and $z_{T}=0$, which corresponds to the present time of the universe. The transition would takes place in past if $\zeta_{0}>0.15$ and in future if $0<\zeta_{0}<0.15$.

The result regarding the transition of the universe into accelerated epoch discussed above have been further verified by studying the evolution of DP. The viscous NHDE model gives time-dependent DP which would describe the phase transition. We have obtained $q$ in terms of $a$ and $z$. The variation of $q$ with $a$ has been shown in Fig. 2a, b with varying $\zeta_{0}$ and constant model parameters, and varying model parameters and constant $\zeta_{0}$, respectively. The evolution of DP shows that the transition from decelerated to accelerated epoch occurs at the present time, corresponding to $q=0$ if $\zeta_{0}=0.15$. The transition would be in recent past, corresponds to $q<0$ at present $\zeta_{0}>0.15$ and the transition into accelerating epoch will be in the future, corresponds to $q>0$ at present if $0<\zeta_{0}<0.15$.

As the model predicts the late time acceleration, we have analyzed the model using statefinder parameter and $\mathrm{Om}$ diagnostic to distinguished it from other DE models especially from $\Lambda C D M$ model. The evolution of the viscous NHDE model in the $\{r, s\}$ plane is shown in Fig. 3a with different values of $\zeta_{0}$ with constant $\alpha$ and $\beta$. It shows that the evolution of $\{r, s\}$ parameter is in such a way that $r<1, s>0$, a feature of quintessence model where as $r>1, s<0$ corresponds to the Chaplygin gas model. In both models, the trajectories are coinciding with each other for any value of $\zeta_{0}$. The viscous NHDE model behaving quintessence and Chaplygin gas models in early time for different $\zeta_{0}$ untimely approaches to $\Lambda C D M$ model in late time. We have also discussed the evolutionary behavior of $\{r, q\}$ to discriminate the viscous NHDE model. The trajectory of $\{r, q\}$ has been plotted in Fig. $3 \mathrm{~b}$ which shows the phase transition from decelerated to accelerated phase. If $0<\zeta_{0} \leq 0.57$, the transition takes place from quintessence region and approaches to $S S$ model in late time as $\Lambda C D M$ model approaches from SCDM. However, if $\zeta_{0}>0.57$, the transition starts from Chaplygin gas model and approaches to $S S$ model in late time. Both the trajectories in $Q$ model and $C G$ model are coinciding on each other for any value of $\zeta_{0}$.

A study of $\mathrm{Om}$ diagnostic of viscous NHDE model has been carried out in Fig. 5a for different values of $\zeta_{0}$ and fixed $\alpha$ and $\beta$. The trajectory shows that if $0<\zeta_{0} \leq 0.57$, the $\operatorname{Om}(z)$ trajectory shows the negative slope which means viscous NHDE behaves like quintessence and if $\zeta_{0}>0.57$, the positive slope of the $\operatorname{Om}(z)$ trajectory is observed, i.e., the viscous NHDE behaves like phantom. In future as $z \rightarrow-1$, the $\operatorname{Om}(z)$ becomes constant, i.e, it may approach to $\Lambda C D M$ model.

The above discussion shows that effect of bulk viscous coefficient on NHDE model with different values of $\zeta_{0}$. We have also discussed the viscous NHDE model with varying model parameters $\alpha$ and $\beta$ taking fixed $\zeta_{0}$. The trajectory for $q$ versus $a$ as shown in Fig. 2b shows that the transition takes place from decelerated to accelerated phase in future for any values of $\alpha$ and $\beta$ and approaches to $q=-1$ in late-time. The trajectory for $\{r, s\}$ and $\{r, q\}$ have also been plotted respectively in Fig. 4a, b for different values of $\alpha$ and $\beta$ with fixed value of $\zeta_{0}$. The $\{r, s\}$ trajectory as shown in Fig. 4a shows that the trajectory starts from the quintessence region, even though some starts from the vicinity of $S C D M$ and approaches to $\Lambda C D M$ in late-time. The signature change of $q$ from positive to negative has been observed in $r-q$ plane as shown in Fig. $4 \mathrm{~b}$. The viscous NHDE model approaches to $S S$ model in late-time as $\Lambda C D M$ does. The $O m$ trajectory has been plotted in Fig. $5 \mathrm{~b}$ for different values of $\alpha$ and $\beta$ for fixed $\zeta_{0}$. This trajectory only shows the negative curvature which imply that the viscous NHDE behaves like quintessence.

From the above discussion with constant bulk viscous coefficient, we find that the constant $\zeta_{0}$ (or cosmological parameters $\alpha$ and $\beta$ ) play important roles in the evolution of the universe i.e., they both determine the evolutionary behavior as well as the ultimate fate of the universe.

In second viscous NHDE model we have assumed $\zeta=$ $\zeta_{1} H$. The solution of this model is similar to the non-viscous NHDE one. We have obtained power-law form of scale factor which gives constant values of DP and statefinder pairs.

In last case we have taken the most general form of bulk viscous coefficient $\zeta=\zeta_{0}+\zeta_{1} H$. The solution of this model is similar to the constant bulk viscous coefficient $\zeta_{0}$. The effect of both non-zero values of $\zeta_{0}$ and $\zeta_{1}$ have been discussed. We have obtained exponential scale factor which gives time-dependent DP and statefinder pairs. The transition from decelerated to accelerated epoch has been discussed. As $\left(t-t_{0}\right) \rightarrow 0$, the scale factor asymptotically gives the power-law which shows that the model decelerates in early time and accelerates in late-time. As $\left(t-t_{0}\right) \rightarrow \infty$, the model corresponds to de Sitter like. The transition scale factor and 
hence corresponding transition redshift has been calculated. If $\left(\zeta_{0}+\zeta_{1}\right)=0.15$, the transition from decelerated to accelerated phase occurs at $a_{T}=1$ or $z_{T}=0$, i.e., at present time of the universe. Figure 6 shows that evolution of the scale factor with $\left(t-t_{0}\right)$. If $0<\left(\zeta_{0}+\zeta_{1}\right)<0.15$, the scale factor has earlier deceleration phase followed by an acceleration phase in later stage of the evolution. For $\left(\zeta_{0}+\zeta_{1}\right)>0.15$, the transition takes place in past of the universe and the scale factor increases with accelerated rate forever.

The DP is time-dependent which shows phase transition from decelerated to accelerated phase. The DP has been written in terms of scale factor or redshift. We have calculated the present value $q_{0}$ which gives $\zeta_{0}+\zeta_{1}=0.15$. This shows that the transition into acceleration phase occurs at present time. If $\left(\zeta_{0}+\zeta_{1}\right)>0.15, q_{0}<0$, i.e., the universe is in accelerating phase and it entered this epoch at an early stage. If $\left(\zeta_{0}+\zeta_{1}\right)<0.15, q_{0}>0$, i.e., the universe is in decelerating epoch and it will be entered into the accelerated phase in future. We have plotted $q$ versus $a$ for different values of $\left(\zeta_{0}, \zeta_{1}\right)$ with fixed model parameters and others as shown in Fig. 7a. The Fig. 7b plots the $q-a$ for different models parameters $\alpha, \beta$ with fixed $\zeta_{0}, \zeta_{1}$ and others.

Figure 8a shows the $r-s$ trajectory in $r-s$ plane for different values of $\zeta_{0}$ and $\zeta_{1}$ with constant model parameters and others. The model behaviors to $Q$ models for $0<\left(\zeta_{0}+\zeta_{1}\right) \leq 0.57$ and $C G$ models for $\left(\zeta_{0}+\zeta_{1}\right)>0.57$. The trajectories in $Q$-model and $C G$ model both converge to the $\Lambda C D M$ model in late time of evolution. Figure $8 \mathrm{~b}$ plots the trajectory of $q-r$ for different values of $\left(\zeta_{0}, \zeta_{1}\right)$ with constant model parameters and others. The DP changes its sign from positive to negative with respect to time which shows the phase transition of the universe from deceleration to acceleration. In beginning this model behaves different from the $\Lambda C D M$ but in future it behaves same as $\Lambda C D M$ which converges to $S S$ model in late-time. Thus, the variable viscous NHDE model is compatible with both $Q$ and $C G$ model. Figure 9a, b plot the trajectories of $r-s$ and $r-q$ for different model parameters $(\alpha, \beta)$ with fixed $\zeta_{0}, \zeta_{1}$ and others. In Fig. 9a, we have analysed that for this fixed value of $\zeta_{0}$ and $\zeta_{1}$ the $\{r, s\}$ trajectories are lying in the region corresponds to $r<1, s>0$ which shows that our model is similar to the $Q$ model. Figure $9 \mathrm{~b}$ shows that this model is also able to explain the phase transition of the universe. It also starts from the neighbourhood of the SCDM model for some values of $\alpha$ and $\beta$. In future the variable viscous NHDE model approaches to the $S S$ model same as the $\Lambda C D M$ and $Q$ models. Thus the viscous NHDE model is compatible with the $\Lambda C D M$ and $Q$ models.

We conclude that the trajectory of $s-r$ and $q-r$ suggest a different behavior as compare to Ricci dark energy done by Feng [16] where it was found that the $s-r$ trajectory is a vertical segment, i.e., $s$ is constant during the evolution of the universe. The trajectory in our viscous NHDE model is mostly confined a parabolic curve and approaches to $\{r, s\}=$ $\{1,0\}$ in $s-r$ plane and $\{r, q\}=\{1,-1\}$ in $q-r$ plane.

From $\mathrm{Om}$ diagnostic we find that the trajectory represents the negative curvature, i.e, viscous NHDE behaves as a quintessence for $0<\left(\zeta_{0}+\zeta_{1}\right) \leq 0.57$ and it shows the positive curvature, i.e., the viscous DE behaves as phantom, for $\left(\zeta_{0}+\zeta_{1}\right)>0.57$, which is graphically represented by Fig. 10a. We have also concluded that as $z \rightarrow-1$, we get the constant value of $\mathrm{Om}$, which corresponds to $\Lambda C D M$ model. However, plot of $\mathrm{Om}$ as shown in Fig. 10b for different model parameters with constant $\zeta_{o}$ and $\zeta_{1}$ reveal that there is always negative curvature for any values of model parameters. This shows that the viscous NHDE behaviors similar to quintessence.

In concluding remarks, let us compare our work with respect to the earlier studied in this direction. Feng and $\mathrm{Li}$ [56] who investigated the viscous Ricci DE model by assuming bulk viscous coefficient proportional to the velocity vector of the fluid. Chattopadhyay [55] reported a study on modified Chaplygin gas based reconstructed scheme for extended HDE in the presence of bulk viscosity. In comparison to the said work, the present work lies not only in its choice of different bulk viscous coefficient but also in its different approach to discuss the evolution of the universe. The present viscous NHDE model successfully describes the present accelerated epoch. The $\Lambda C D M$ model is attainable by present model. The NHDE model behaves quintessence model and Chaplygin gas model inn early time due to viscous effect. However, it behaves only quintessence if we consider the model parameters with fixed viscous coefficient. Our work implies the theoretical basis for future observations to constraint the viscous NHDE.

Acknowledgements The authors express their sincere thank to the referee for his suggestions to improve the manuscript. One of the author MS sincerely acknowledges the University Grant Commission, which provided the Senior Research Fellowship under UGC-NET Scholarship.

Open Access This article is distributed under the terms of the Creative Commons Attribution 4.0 International License (http://creativecomm ons.org/licenses/by/4.0/), which permits unrestricted use, distribution, and reproduction in any medium, provided you give appropriate credit to the original author(s) and the source, provide a link to the Creative Commons license, and indicate if changes were made. Funded by SCOAP . $^{3}$.

\section{References}

1. A.G. Riess et al., Astron. J. 116, 1009 (1998)

2. S. Perlmutter et al., Nature 391, 51 (1998)

3. S. Perlmutter et al., Astrophys. J. 517, 565 (1999)

4. D.N. Spergel et al., Astrophys. J. Suppl. Ser. 148, 175 (2003)

5. D.N. Spergel et al., Astrophys. J. Suppl. Ser. 170, 377 (2007)

6. M. Tegmark et al., Phys. Rev. D 69, 103501 (2004)

7. M. Tegmark et al., Astrophys. J. 606, 702 (2004)

8. S.M. Carroll, Living Rev. Relativ. 4, 1 (2001) 
9. S. Weinberg, Rev. Mod. Phys. 61, 1 (1989)

10. P.J.E. Peebles, Rev. Mod. Phys. 75, 559 (2003)

11. E.J. Copeland, M. Sami, S. Tsujikawa, Int. J. Mod. Phys. D 15, $1753(2006)$

12. P.J.E. Peebles, B. Ratra, Astrophys. J. 325, L17 (1988)

13. M.S. Turner, Int. J. Mod. Phys. A 17, 180 (2002)

14. R.R. Caldwell, M. Kamionkowski, N.N. Weinberg, Phys. Rev. Lett. 91, 071301 (2003)

15. T. Padmanabhan, T.R. Chaudhary, Phys. Rev. D 66, 081301 (2002)

16. B. Feng et al., Phys. Lett. B 607, 35 (2005)

17. S.D. Hsu, Phys. Lett. B 594, 13 (2004)

18. M. Li, Phys. Lett. B 603, 1 (2004)

19. R.G. Cai, Phys. Lett. B 657, 228 (2007)

20. H. Wei, R.G. Cai, Phys. Lett. B 660, 113 (2008)

21. A.A. Starobinsky, Phys. Lett. B 91, 99 (1980)

22. R. Kerner, Gen. Relativ. Gravit. 14, 453 (1982)

23. G. t'Hooft, arXiv:gr-qc/9310026

24. L. Susskind, J. Math. Phys. 36, 6377 (1995)

25. R. Bousso, Class. Quantum Gravity 17, 997 (2000)

26. A.G. Cohen, D.B. Kaplan, A.E. Nelson, Phys. Rev. Lett. 82, 4971 (1999)

27. H. Wei, S.N. Zhang, arXiv:0707.2129

28. C. Gao, F. Wu, X. Chen, Phys. Rev. D 79, 043511 (2009)

29. L.N. Granda, A. Oliveros, Phys. Lett. B 669, 275 (2008)

30. L.N. Granda, A. Oliveros, Phys. Lett. B 671, 199 (2009)

31. K. Karami, J. Fehri, Phy. Lett. B 684, 61 (2010)

32. M. Malekjani, A. Khodam-Mohammadi, N. Nazari-Pooya, arXiv: 1011.4805 [gr-qc]

33. M. Sharif, A. Jawad, arXiv:1212.0129 [gr-qc]

34. U. Debnath, S. Chattopadhyay, arXiv:1102.0091 [physics.gen-ph]

35. Y. Wang, L. Xu, Phys. Rev. D 81, 083523 (2010)

36. A. Oliveros, M.A. Acero, Astrophys. Space Sci. 357, 12 (2015)

37. V. Sahni et al., JETP Lett. 77, 201 (2003)

38. U. Alam et al., Mon. Not. R. Astron. Soc. 344, 1057 (2003)

39. V. Sahni, A. Shafieloo, A.A. Starobinsky, Phys. Rev. D 78, 103502 (2008)

40. C. Eckart, Phys. Rev. D 58, 919 (1940)

41. W. Israel, J.M. Stewart, Phys. Lett. A 58, 213 (1976)

42. X.H. Meng, J. Ren, M.G. Hu, Commun. Theor. Phys. 47, 379 (2007)

43. X.H. Meng, X. Duo, Commun. Theor. Phys. 52, 377 (2009)

44. J. Ren, X.H. Meng, Phys. Lett. B 633, 1 (2006)

45. M.G. Hu, X.H. Meng, Phys. Lett. B 635, (2006).
46. C.P. Singh, S. Kumar, A. Pradhan, Class. Quantum Gravity 24, 455 (2007)

47. C.P. Singh, Pramana J. Phys. 71, 33 (2009)

48. C.P. Singh, Mod. Phys. Lett. A 27, 1250070 (2012)

49. C.P. Singh, P. Kumar, Eur. Phys. J. C 74, 3070 (2014)

50. P. Kumar, C.P. Singh, Astrophys. Space Sci. 357, 120 (2015)

51. I. Brevik et al., Int. J. Mod. Phys. D 26, 1730024 (2017)

52. B.D. Normann, I. Brevik, Mod. Phys. Lett. A 32, 1750026 (2017)

53. B.D. Normann, I. Brevik, Entropy 18, 215 (2016)

54. M. Jamil, M.U. Farooq, Int. J. Theor. Phys. 49, 42 (2010)

55. S. Chattopadhyay, Int. J. Mod. Phys. D 26, 1750042 (2017)

56. C.-J. Feng, X.-Z. Li, Phys. Lett. B 680, 355 (2009)

57. C.P. Singh, P. Kumar, Astrophys. Space Sci. 361, 157 (2016)

58. I. Brevik, O. Gorbunova, Y.A. Shaido, Int. J. Mod. Phys. D 14, 1899 (2005)

59. I. Brevik, O. Gorbunova, Eur. Phys. J. C 56, 425 (2008)

60. I. Brevik, O. Gorbunova, D.S. Gomez, Gen. Relativ. Gravit. 42, $1513(2010)$

61. W. Zimdahl, D. Pavon, Phys. Lett. B 521, 133 (2001)

62. W. Zimdahl, D. Pavon, Gen. Relativ. Gravit. 35, 413 (2003)

63. L.P. Chimento, A.S. Jakubi, D. Pavon, W. Zimdahl, Phys. Rev. D 67, 083513 (2003)

64. R. Maartens, Class. Quantum Gravity 12, 1455 (1995)

65. A. Avelino, U. Nucamendi, J. Cosmol. Astropart. Phys. 08, 009 (2010)

66. C.J. Feng, Phys. Lett. B 670, 231 (2008)

67. B. Wu, S. Li, M.H. Fu, J. He, Gen. Relativ. Grav. 39, 653 (2007)

68. T.K. Mathew, J. Suresh, D. Divakaran, Int. J. Mod. Phys. D 22, 1350056 (2013)

69. Z.G. Haung, X.M. Song, H.Q. Lu, W. Fang, Astrophys. Space Sci. 315, 175 (2008)

70. B. Wang, Y. Gong, E. Abdalla, Phys. Rev. D 74, 083520 (2006)

71. M.L. Tong, Y. Zhang, Phys. Rev. D 80, 023503 (2009)

72. J.B. Lu, L.X. Xu, Int. J. Mod. Phys. D 18, 1741 (2009)

73. Z.G. Huang, H.Q. Lu, K. Zhang, Astrophys. Space Sci. 331, 331 (2011) 